Contributions to Game Theory and Management, XIII, 95-120

\title{
Dynamic Cooperative Network Games with Pairwise Interactions ${ }^{\star}$
}

\author{
Mariia A. Bulgakova \\ St.Petersburg State University, \\ Faculty of Applied Mathematics and Control Processes, \\ Universitetskiy prosp. 35, Peterhof, 198504, Russia \\ E-mail: mari_bulgakova@mail.ru
}

\begin{abstract}
This article is an overview of results obtained in the field of dynamic network games with pairwise interaction. The paper provides a summary and analysis of works related to two-stage and multistage nonzero-sum games based on pairwise interaction. The meaning of pairwise interaction is to consider the game as a family of games occurring on a network between pairs of players (vertices of a graph) connected to each other by an edge. The network can be set or formed in the first stage. In the paper, solutions of cooperative pairwise interaction games are also considered.
\end{abstract}

Keywords: cooperative games, network games, dynamic games, the Shapley value, pairwise interactions, time-consistency.

\section{Introduction}

Cooperative network games is an important part of modern game theory. Networks illustrate connections between players and their ability to communicate in coalitions. For the first time in the game theory literature, a non-cooperative form of pairwise interaction in a network was considered in (Dyer and Mohanaraj, 2011) meaning direct interactions between network neighbors. But cooperative approach is quite natural and moreover beneficial for players as it provides them with a better outcome rather than noncooperative behavior. First cooperative interpretation of pairwise interaction games was in (Bulgakova and Petrosyan, 2015). Solutions of network games with pairwise interactions were examined in detail in (Bulgakova, 2019).

Also of interest are solutions for games with pairwise interaction on specific networks. The geometric structure of the network and symmetry make it possible to simplify some complex formulas and get their simple explicit form (Petrosyan, Sedakov and Bulgakova, 2018; Bulgakova and Petrosyan, 2019a).

When cooperative behavior is investigated, it is important that players follow a cooperative agreement during the whole course of the game. If a solution of the cooperative game is time consistent, players have no reason to deviate from the accepted agreement. Conditions for strongly time-consistence of core in 3-person games with pairwise interactions was studied in (Bulgakova and Petrosyan, 2016). Also, time consistent cooperative solutions for multistage games with special payoffs wad considered in (Kuzyutin and Nikitina, 2017).

Sometimes it is difficult to determine the explicit form of the core and decide on its stability, in this case, subsets of the core are used, which can be found in various ways. For example, as in (Petrosyan and Pankratova, 2018). Also, a special subset of

\footnotetext{
${ }^{\star}$ This work was supported by the Russian Science Foundation (project No.17-11-01079).
} 
the core, the IDP-core, was considered in (Petrosian, Gromova and Pogozhev, 2018; Wolf, Zakharov and Petrosian, 2017). The adaptation of the IDP-core to games with pairwise interaction was carried out in (Bulgakova and Petrosyan, 2019b).

In this paper, we analyse and compare the results in the field of cooperative network games with pairwise interaction.

\section{Two-Stage Network Games with Pairwise Interactions}

Consider a model of two stage cooperative network games with pairwise interactions (Dyer and Mohanaraj, 2011), when network is not given. So, players should form a network on the first stage. These game was studied in (Bulgakova and Petrosyan, 2015). Let us explain the model.

Let $N$ be a finite set of players, which can make decisions in two stages, $|N|=$ $n \geq 2$. Denote as $z$ stage of game. The game started in stage $z_{1}$, where every player $i \in N$ choose his behavior $b_{i}^{1}=\left(b_{i 1}^{1}, \ldots, b_{i n}^{1}\right)-n$-dimensional vector with offers for connections to other players (Petrosyan, Sedakov and Bochkarev, 2013).

We will use following notations: $M_{i} \subseteq N \backslash\{i\}$ - those players, whom player $i \in N$ can offer a connection, wherein $a_{i} \in\{0, \ldots, n-1\}$ is maximal number of connections for player $i$, which he can maintain simultaneously. If $M_{i}=N \backslash\{i\}$, it means, that player $i$ can offer connections for all players. In case, if $a_{i}=n-1$, player $i$ can maintain any number of connections.

For every behavior $b_{i}^{1}$ there exists such subset of realized offers $Q_{i} \subset M_{i}$, which satisfy following restrictions

$$
b_{i j}^{1}=\left\{\begin{array}{l}
1, \text { if } j \in Q_{i}, \\
0, \text { other }
\end{array}\right.
$$

with additional condition

$$
\sum_{j \in N} b_{i j}^{1} \leq a_{i}
$$

Condition (2) means, that number of possible connections is restricted for every player. Also, obviously, $\left|Q_{i}\right| \leq a_{i}$.

Connection $i j$ is formed if and only if, $b_{i j}^{1}=b_{j i}^{1}=1$. Formed connections $i j$ create edges of network $g$, where vertex are players, i.e., if $b_{i j}^{1}=b_{j i}^{1}=1$, then there is an edge between $i$ and $j$ in network $g$.

Denote by $N_{i}(g)$ or simply $N_{i}$ neighbours of player $i$ in network $g$, i.e. $N_{i}(g)=$ $\{j \in N \backslash\{i\}: i j \in g\}$. As result of the first stage we have network $g\left(b_{1}^{1}, \ldots, b_{n}^{1}\right)$. After network is formed players move to stage $z_{2}(g)$, which depend on network. On second stage $z_{2}(g)$, neighbours on network play pairwise in simultaneous bimatrix games, after that players get their payoffs and the game ends. In other words, we have two stage game $\Gamma_{z_{1}}(g)$, which is special case of multistage nonzero-sum games. In considered case strategy of player is a rule, which for every player determine his set of neighbours on the first stage, namely vector $b_{i}^{1}$, and his behavior in every bimatrix game on second stage corresponding to network, which is formed on the first stage $-b_{i}^{2}$. Denote by $u_{i}=\left(b_{i}^{1}, b_{i}^{2}\right), i \in N$, strategy of player $i$ in two-stage game $\Gamma_{z_{1}}(g)$. Calculate payoff of player $i$ as $h_{i}\left(z_{2}\right)$, where $\left(z_{1}, z_{2}\right)$ is a path, realized by strategy profile $u=\left(u_{1}(\cdot), \ldots, u_{n}(\cdot)\right)$ in game $\Gamma_{z_{1}}(g)$. Because on the first stage players do not get their payoffs, payoff function in game $\Gamma_{z_{1}}(g)$ with starting position $z_{1}$ is determined by following expression:

$$
K_{i}\left(z_{1} ; u\right)=K_{i}\left(z_{1} ; u_{i}(\cdot), \ldots, u_{n}(\cdot)\right)=h_{i}\left(z_{2}\right) .
$$




\subsection{Characteristic Function}

On second stage game represents a set of pairwise simultaneous bimatrix games $\left\{\gamma_{i j}\right\}$ between neighbours in network. Namely, let $i \in N, j \in N_{i}$. Then player $i$ (the first player) play with player $j$ (the second player)in bimatrix game $\gamma_{i j}$ with payoff matrices $A_{i j}$ and $C_{i j}$ of players $i$ and $j$ respectively.

$$
\begin{gathered}
A_{i j}=\left(\begin{array}{cccc}
a_{11}^{i j} & a_{12}^{i j} & \cdots & a_{1 k}^{i j} \\
a_{21}^{i j} & a_{22}^{i j} & \cdots & a_{2 k}^{i j} \\
\vdots & \vdots & \ddots & \vdots \\
a_{m 1}^{i j} & a_{m 2}^{i j} & \cdots & a_{m k}^{i j}
\end{array}\right) \\
C_{i j}=\left(\begin{array}{cccc}
c_{11}^{i j} & c_{12}^{i j} & \cdots & c_{1 k}^{i j} \\
c_{21}^{i j} & c_{22}^{i j} & \cdots & c_{2 k}^{i j} \\
\vdots & \vdots & \ddots & \vdots \\
c_{m 1}^{i j} & c_{m 2}^{i j} & \cdots & c_{m k}^{i j}
\end{array}\right) \\
a_{p l} \geq 0, \quad c_{p l} \geq 0, \quad p=1, \ldots, m, \quad l=1, \ldots, k .
\end{gathered}
$$

Constants $m$ and $k$ coincide for all $i$ and $j$. When we have game $\gamma_{j i}$, i.e. player $i$ is the second player, he has payoff matrix $C_{j i}=A_{i j}^{T}$, and player $j$ has matrix $A_{j i}=C_{i j}^{T}$. Denote by $\Gamma_{z_{2}}^{S}(g)$ subgame of game $\Gamma$, which takes place on second stage $z_{2}$. Consider this game in cooperative form. Find characteristic function for every subset (coalition) $S \subset N$ as lower (maxmin) value for two person zero-sum game of coalition $S$ and coalition $N \backslash S$, based on game $\Gamma_{z_{2}}^{S}(g)$. Payoff of coalition $S$ is sum payoffs of players from coalition $S$. Super-additivity of characteristic function follows from its definition. Note, that

$$
\begin{aligned}
& w_{i j}^{i}=\max _{p} \min _{\ell} a_{p \ell}^{i j}, \quad p=1, \ldots, m ; \quad \ell=1, \ldots, k, \\
& w_{i j}^{j}=\max _{\ell} \min _{p} c_{p \ell}^{j i}, \quad p=1, \ldots, m ; \quad \ell=1, \ldots, k .
\end{aligned}
$$

and $v\left(z_{2} ; S\right), S \subset N$, is lower value of zero-sum game $\Gamma_{z_{2}}^{S}(g)$.

Theorem 1 (Bulgakova, 2019). Function $v\left(z_{2} ; S\right)$ is determined by following expressions:

$$
\begin{gathered}
v\left(z_{2} ;\{\emptyset\}\right)=0, \\
v\left(z_{2} ;\{i\}\right)=\sum_{j \in N_{i}} w_{i j}^{i}, \\
v\left(z_{2} ; S\right)=\frac{1}{2} \sum_{i \in S} \sum_{j \in N_{i} \cap S} \max _{p, \ell}\left(a_{p \ell}^{i j}+c_{p \ell}^{i j}\right)+\sum_{i \in S} \sum_{k \in N_{i} \backslash S} w_{i k}^{i}, S \subset N, \\
v\left(z_{2} ; N\right)=\frac{1}{2} \sum_{i \in N} \sum_{j \in N_{i}} \max _{p, \ell}\left(a_{p \ell}^{i j}+c_{p \ell}^{i j}\right) .
\end{gathered}
$$


Consider a cooperative form of two-stage game $\Gamma_{z_{1}}(g)$. Suppose, that players chose strategies $\bar{u}_{i}, i \in N$, that maximize their total payoff in game $\Gamma_{z_{1}}(g)$, i. e.

$$
\sum_{i \in N} K_{i}\left(z_{1} ; \bar{u}_{1}, \ldots, \bar{u}_{n}\right)=\max _{u} \sum_{i \in N} K_{i}\left(z_{1} ; u_{1}, \ldots, u_{n}\right) .
$$

Strategy profile $\bar{u}=\left(\bar{u}_{1}, \ldots, \bar{u}_{n}\right)$ we will call cooperative behavior, and corresponding path $\left(\bar{z}_{1}, \bar{z}_{2}\right)$ - cooperative path.

As previously, for coalition $S \subseteq N$ define characteristic function $v\left(\bar{z}_{1} ; S\right)$ as maxmin value in two person zero-sum game between coalition $S$ (maximizing player) and coalition $N \backslash S$ (minimizing player). For minimazing player the best way of behavor is to not create all the connections with maximizing player (because of positive payoffs for each connection). Payoff of coalition $S$ is sum of payoffs of its members.

Denote by $v\left(z_{1} ; S\right), S \subset N$, lower value of zero-sum game $\Gamma_{z_{1}}(g)$.

Theorem 2. Function $v\left(z_{1} ; S\right)$ is defined in following way:

$$
\begin{gathered}
v\left(\bar{z}_{1} ;\{i\}\right)=0, \quad v\left(\bar{z}_{1} ; \emptyset\right)=0, \\
v\left(\bar{z}_{1} ; S\right)=\frac{1}{2} \sum_{i \in S} \sum_{j \in N_{i}(g) \cap S} \max _{p, \ell}\left(a_{p \ell}^{i j}+c_{p \ell}^{i j}\right), \quad S \subset N, \\
v\left(\bar{z}_{1} ; N\right)=v\left(\bar{z}_{2} ; N\right)=\frac{1}{2} \sum_{i \in N} \sum_{j \in N_{i}} \max _{p, \ell}\left(a_{p \ell}^{i j}+c_{p \ell}^{i j}\right) .
\end{gathered}
$$

\subsection{Convex Game}

Definition 1. Characteristic function is called supermodular and corresponding game called convex (Shapley, 1971), if for any coalition $X \subset N$ and $Y \subset N$ holds inequality:

$$
v(X \cup Y) \geq v(X)+v(Y)-v(X \cap Y) .
$$

Theorem 3 (Bulgakova, 2019). In subgame $\Gamma_{z_{2}}^{S}(g)$ characteristic function (8)(10) is supermodular.

This property guarantees non-emptiness of the core and that the Shapley value belongs to the core.

\subsection{Star-Network}

Consider a specific structure of network, and solution of cooperative game on this network. We will study network, which contains $n$ players, where player 1 is central player with $n-1$ connections, and all other $n-1$ players are connected by edge only with a central player.

The Shapley Value. Denote the set of imputations by

$$
\mathcal{M}\left[v\left(\bar{z}_{t}\right)\right]=\left\{x=\left(x_{1}, \ldots, x_{n}\right): \sum_{i=1}^{n} x_{i}=v\left(\bar{z}_{t} ; N\right), x_{i} \geq v\left(\bar{z}_{t} ;\{i\}\right), i \in N\right\} .
$$


Consider as a solution of star-network the Shapley value (Shapley, 1953) and denote it as $\varphi\left[v\left(\bar{z}_{t}\right)\right]=\left(\varphi_{1}\left[v\left(\bar{z}_{t}\right)\right], \ldots, \varphi_{n}\left[v\left(\bar{z}_{t}\right)\right]\right), t=1,2$, where for every player $i \in N$

$$
\varphi_{i}\left[v\left(\bar{z}_{t}\right)\right]=\sum_{\substack{S \subseteq N, i \in S}} \frac{(|S|-1) !(n-|S|) !}{n !}\left[v\left(\bar{z}_{t} ; S\right)-v\left(\bar{z}_{t} ; S \backslash\{i\}\right)\right] .
$$

We say that the cooperative solution $\mathcal{M}\left[v\left(\bar{z}_{1}\right)\right]$ in the two-stage game is time consistent if for any imputation $\xi\left[v\left(\bar{z}_{1}\right)\right] \in \mathcal{M}\left[v\left(\bar{z}_{1}\right)\right]$ there exists an imputation $\xi\left[v\left(\bar{z}_{2}\right)\right] \in \mathcal{M}\left[v\left(\bar{z}_{2}\right)\right]$ such that

$$
\xi\left[v\left(\bar{z}_{1}\right)\right]=\xi\left[v\left(\bar{z}_{2}\right)\right]
$$

Thus the Shapley value $\varphi\left[v\left(\bar{z}_{1}\right)\right]$ is a time consistent cooperative solution if

$$
\varphi\left[v\left(\bar{z}_{1}\right)\right]=\varphi\left[v\left(\bar{z}_{2}\right)\right],
$$

otherwise the Shapley value is time inconsistent.

One has to mention that the cooperative version of the game has been developed for a general case of pairwise interactions when any (feasible) network can be formed at the first stage of the game. For this general case, we have found analytical expressions for the characteristic functions (8), (11) which are used to calculate the Shapley value by (15) and verify the corresponding time consistency condition 17 . Since the calculation of the Shapley value is a challenging task for a large number of players and an arbitrary network, in the following we will show how to simplify formula 15 for a network of a special type - a star. This type of the network will provide us with an analytical expression for the Shapley value which is much easier to interpret and analyze. Nevertheless, expressions (8), (11), (15) can help us to find the Shapley value for any network at least numerically. From (15) we note that to compute a component of the Shapley value, we need to list all $2^{n}$ subsets of the player set $N$; moreover for large networks we may be limited in computational capabilities to calculate $n$ ! as this number can be extremely large. In contrast, for a star network we will need only $O(n)$ calculations and will not need to list all subsets of $N$.

Because the Shapley value belongs to the core in subgame $\Gamma_{z_{2}}^{S}(g)$, then its significance in this class of problems increases.

Now formalize construction of star-network on the first stage of game. Suppose following, let $M_{1}=N \backslash\{1\}, a_{1}=n-1$ and $M_{i}=\{1\}, a_{i}=1$ for $i \neq 1$. To maximize total payoff, players should chose on the first stage following behaviors:

$$
b_{i}^{1}=\left\{\begin{array}{l}
(0,1, \ldots, 1), i=1, \\
(1,0, \ldots, 0), i \neq 1 .
\end{array}\right.
$$

Behavior (18) formed a star network on the first stage with central player 1, (fig. 1 ), where $\left|N_{1}\right|=n-1$ and $\left|N_{i}\right|=1, i \neq 1$. For star-network calculate the characteristic function in view of specific structure of network. Transform expressions (8-10) corresponding to a star-network:

$$
\begin{gathered}
v\left(z_{2} ;\{\emptyset\}\right)=0, \\
v\left(z_{2} ;\{i\}\right)=\left\{\begin{array}{l}
\sum_{j \neq i} w_{1 j}^{1}, i=1, \\
w_{1 i}^{i}, \quad i \neq 1 .
\end{array}\right.
\end{gathered}
$$




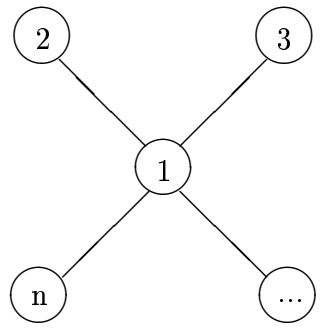

Fig. 1. Star-network

$$
v\left(z_{2} ; S\right)= \begin{cases}\sum_{j \in N_{1} \cap S} \max _{p, \ell}\left(a_{p \ell}^{1 j}+c_{p \ell}^{1 j}\right)+\sum_{k \in N_{1} \backslash S} w_{1 k}^{1}, S \subset N, i \in S, \\ 0, & i \notin S .\end{cases}
$$

Similarly, we transform the expressions (11)-(13)

$$
\begin{gathered}
v\left(\bar{z}_{1} ;\{i\}\right)=0, \quad v\left(\bar{z}_{1} ; \emptyset\right)=0, \\
v\left(\bar{z}_{1} ; S\right)=\left\{\begin{array}{lr}
\sum_{j \in N_{1} \cap S} \max _{p, \ell}\left(a_{p \ell}^{1 j}+c_{p \ell}^{1 j}\right), S \subset N, i \in S, \\
0,
\end{array}\right.
\end{gathered}
$$

The network has central symmetry and formula (15) can be simplified.

Proposition 1 (Petrosyan, Sedakov and Bulgakova, 2018). For star-network with central player 1 , components of the Shapley value $\varphi\left[v\left(\bar{z}_{t}\right)\right], t=1,2$, has the form:

$$
\varphi_{i}\left[v\left(\bar{z}_{t}\right)\right]= \begin{cases}\frac{1}{2}\left[v\left(\bar{z}_{t} ;\{1\}\right)+\sum_{j \neq 1}\left(m_{1 j}-v\left(\bar{z}_{t} ;\{j\}\right)\right)\right], & i=1, \\ \frac{1}{2}\left[v\left(\bar{z}_{t} ;\{i\}\right)+m_{1 i}-w_{1 i}^{1}\right], & i \neq 1 .\end{cases}
$$

where

$$
m_{i j}= \begin{cases}\max _{p=1, \ldots, m}\left(a_{p \ell}^{i j}+b_{p \ell}^{i j}\right), & \text { if } i \text { and } j \text { are neighbors }, \\ \ell=1, \ldots, k & \text { otherwise. }\end{cases}
$$

Time-Consistency of the Shapley Value in a Star-Network Consider timeconsistency of Shapley value in a star-network. Remind that the Shapley value $\varphi\left[v\left(\bar{z}_{1}\right)\right]$ is time-consistent cooperative solution, if

$$
\varphi\left[v\left(\bar{z}_{1}\right)\right]=\varphi\left[v\left(\bar{z}_{2}\right)\right] .
$$

Proposition 2 (Petrosyan, Sedakov and Bulgakova, 2018). For star-network with central player 1 , the Shapley value is time-consistent, when $w_{1}^{1 i}=w_{i}^{i 1}$ for any player $i \neq 1$.

Conditions for time-consistency $w_{1}^{1 i}=w_{i}^{i 1}, i \neq 1$, from (2) for star-network can be interpreted in following way: central player 1 and any other player $i \neq 1$ should have similar minimal guaranteed payoff (maxmin value) in bimatrix game $\gamma_{1 i}$ which 
they play as neighbours. If at least for one player $j w_{1}^{1 j} \neq w_{j}^{j 1}$, then the Shapley value will be time-inconsistent. However, in the latter case, a cooperative agreement may be reached at the expense of IDP - imputation distribution procedure. Remind the definition:

Definition 2. Function $\beta^{i}, i \in N$ called imputation distribution procedure (IDP) $x \in \mathcal{M}$ (see Petrosyan and Danilov, 1979), if

$$
x_{i}=\beta_{i}^{1}+\beta_{i}^{2}, \quad i \in N
$$

Proposition 3. For star-network with central player 1 , time-consistent IDP $\beta=$ $\left\{\beta_{i}^{1}, \beta_{i}^{2}\right\}_{i \in N}$ for the Shapley value has the form:

$$
\beta_{i}^{1}= \begin{cases}\frac{1}{2} \sum_{i \neq 1}\left[w_{i 1}^{i}-w_{1 i}^{1}\right], & i=1 \\ \frac{1}{2}\left[w_{1 i}^{1}-w_{i 1}^{i}\right], & i \neq 1\end{cases}
$$

and

$$
\beta_{i}^{2}= \begin{cases}\frac{1}{2} \sum_{i \neq 1}\left[w_{1 i}^{1}+m_{1 i}-w_{i 1}^{i}\right], & i=1 \\ \frac{1}{2}\left[w_{i 1}^{i}+m_{1 i}-w_{1 i}^{1}\right], & i \neq 1\end{cases}
$$

From an economic perspective, it is preferable for players to have a nonnegative IDP, such that decomposing the Shapley value over two game stages, each player receives her nonnegative payments at either of the two game stages. However the IDP of the Shapley value mentioned in the latter proposition can be nonnegative only in case when $w_{1}^{1 i}=w_{i}^{i 1}$ for each $i \neq 1$. This equality results in the time consistency of the Shapley value which has already been discussed.

Three examples below demonstrate that the Shapley value being an imputation in a cooperative two-stage game with pairwise interactions can be both time consistent and time inconsistent despite players' asymmetry in their neighbors. The first example show the time consistency of the Shapley value in an important class of bimatrix games.

Prisoner's Dilemma Consider the case, when $n$ players play the same bimatrix game $\gamma$ with their neighbors, i.e., $A_{i j}=A, C_{i j}=C$ for all $i \in N, j \in N_{i}$ where

$$
A=C^{\mathrm{T}}=\left(\begin{array}{cc}
b & 0 \\
a+b & a
\end{array}\right), \quad 0<a<b .
$$

Here $b$ is the payoff to each connected player if they both "cooperate", while if the both "defect" each of them gets $a$. As an example, one can consider a data transmission problem in a network whose nodes have to "coordinate" their actions trying to achieve certain performance (for example, the number of packets sent, a profit from sending packets).

For the given game using (5), (6), (25) we obtain: $w_{i}^{i j}=w_{j}^{i j}=a$ and

$$
m_{i j}= \begin{cases}2 b, & \text { if } i \text { and } j \text { are neighbors } \\ 0, & \text { otherwise }\end{cases}
$$


for any two players $i, j \in N$.

To find the Shapley value $\varphi\left[v\left(\bar{z}_{2}\right)\right]$, we first determine characteristic function $v\left(\bar{z}_{2} ; S\right)$ for all $S \subseteq N$. Following (8), we obtain

$$
v\left(\bar{z}_{2} ; S\right)= \begin{cases}2 b(n-1), & S=N, \\ 2 b(|S|-1)+(n-|S|) a, & S \subset N, 1 \in S, \\ |S| a, & S \subset N, 1 \notin S, \\ 0, & S=\emptyset .\end{cases}
$$

Using the formula for the Shapley value (24) adapted to the star network, we obtain

$$
\begin{aligned}
\varphi_{1}\left[v\left(\bar{z}_{2}\right)\right] & =\frac{n-1}{2}[a+2 b-a]=b(n-1), \\
\varphi_{i}\left[v\left(\bar{z}_{2}\right)\right] & \left.=\frac{1}{2}[a+2 b-a)\right]=b, \quad i \neq 1 .
\end{aligned}
$$

Similarly, to find the Shapley value $\varphi\left[v\left(\bar{z}_{1}\right)\right]$, we determine characteristic function $v\left(\bar{z}_{1} ; S\right)$ for all $S \subseteq N$. Following (11), we have

$$
v\left(\bar{z}_{1} ; S\right)= \begin{cases}2 b(n-1), & S=N, \\ 2 b(|S|-1), & S \subset N, 1 \in S, \\ 0, & S \subset N, 1 \notin S \quad \text { or } \quad S=\emptyset .\end{cases}
$$

Again, using the formula for the Shapley value (24) adapted to the star network, we get $\varphi\left[v\left(\bar{z}_{1}\right)\right]$ :

$$
\begin{aligned}
& \varphi_{1}\left[v\left(\bar{z}_{1}\right)\right]=\frac{2 b(n-1)}{2}=b(n-1), \\
& \varphi_{i}\left[v\left(\bar{z}_{1}\right)\right]=\frac{2 b}{2}=b, \quad i \neq 1 .
\end{aligned}
$$

Comparing $\varphi\left[v\left(\bar{z}_{1}\right)\right]$ and $\varphi\left[v\left(\bar{z}_{2}\right)\right]$, we note that they coincide and hence the Shapley value is time consistent. What is interesting, this result does not depend upon specific values of $a$ and $b$. The only restriction which has to be imposed is $0<a<b$, and it is natural for this class of games.

The time consistency of the Shapley value can also be shown with the use of Proposition 2 instead of applying direct calculations. Indeed, we see that $w_{i}^{i 1}=$ $w_{1}^{1 i}=a$ for each $i \neq 1$ what implies its time consistency.

Example In the next example we demonstrate the time inconsistency of the Shapley value. Consider a 4-person game with $N=\{1,2,3,4\}$ in which players form a star network under a cooperative agreement (see Fig. 2). Let simultaneous bimatrix games $\gamma_{12}, \gamma_{13}$ and $\gamma_{14}$ be defined by means of the following payoff matrices:

$$
\begin{aligned}
\left(A_{12}, C_{12}\right) & =\begin{array}{l}
(2,2)(3,0) \\
(5,1)(1,2)
\end{array},\left(A_{13}, C_{13}\right)=\begin{array}{l}
(3,1)(4,2) \\
(6,2)(2,3)
\end{array}, \\
\left(A_{14}, C_{14}\right) & =\begin{array}{l}
(1,3)(3,2) \\
(6,6)(4,1)
\end{array}
\end{aligned}
$$

To compute the Shapley values $\varphi\left[v\left(\bar{z}_{1}\right)\right]$ and $\varphi\left[v\left(\bar{z}_{2}\right)\right]$, we use the corresponding formulas (9), (11) for characteristic functions $v\left(\bar{z}_{2} ; \cdot\right)$ and $v\left(\bar{z}_{1} ; \cdot\right)$, respectively, and 


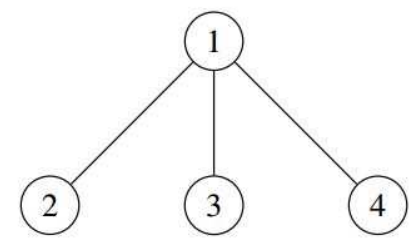

Fig. 2. A star with four players

the simplified formula (24). Hence we get:

$$
\begin{aligned}
& w_{12}^{1}=2, \quad w_{13}^{1}=3, \quad w_{14}^{1}=4, \\
& w_{21}^{2}=1, \quad w_{31}^{3}=2, \quad w_{41}^{4}=3, \\
& m_{12}=6, \quad m_{13}=8, \quad m_{14}=12,
\end{aligned}
$$

and therefore

$$
\begin{aligned}
& v\left(\bar{z}_{1} ;\{1\}\right)=0, v\left(\bar{z}_{2} ;\{1\}\right)=9 \\
& v\left(\bar{z}_{1} ;\{2\}\right)=0, v\left(\bar{z}_{2} ;\{2\}\right)=1 \\
& v\left(\bar{z}_{1} ;\{3\}\right)=0, v\left(\bar{z}_{2} ;\{3\}\right)=2 \\
& v\left(\bar{z}_{1} ;\{4\}\right)=0, v\left(\bar{z}_{2} ;\{4\}\right)=3 \\
& v\left(\bar{z}_{1} ; N\right)=26, v\left(\bar{z}_{2} ; N\right)=26
\end{aligned}
$$

Thus the Shapley values are given by

$$
\begin{aligned}
& \varphi\left[v\left(\bar{z}_{1}\right)\right]=(13,3,4,6), \\
& \varphi\left[v\left(\bar{z}_{2}\right)\right]=(29 / 2,5 / 2,7 / 2,11 / 2) .
\end{aligned}
$$

We observe that the Shapley value $\varphi\left[v\left(\bar{z}_{1}\right)\right]$ in the two-stage game differs from the Shapley value $\varphi\left[v\left(\bar{z}_{2}\right)\right]$ in the one-stage game starting at the second stage. This means time inconsistency of the Shapley value. Since $\varphi_{2}\left[v\left(\bar{z}_{2}\right)\right]=5 / 2<\varphi_{2}\left[v\left(\bar{z}_{1}\right)\right]=$ 3 , player 2 can break the cooperative agreement as she can get less (here we recall that players do not receive payoffs at the network formation stage). Similarly, it is also true for player 3: $\varphi_{3}\left[v\left(\bar{z}_{2}\right)\right]=7 / 2<\varphi_{3}\left[v\left(\bar{z}_{1}\right)\right]=4$ and player $4: \varphi_{4}\left[v\left(\bar{z}_{2}\right)\right]=$ $11 / 2<\varphi_{4}\left[v\left(\bar{z}_{1}\right)\right]=6$. However introducing a time-consistent IDP of the Shapley value $\varphi\left[v\left(\bar{z}_{1}\right)\right]$ over two stages determined with the use of Proposition 3 for the star network, we obtain

$$
\begin{aligned}
& \beta_{1}^{1}=-3 / 2, \quad \beta_{2}^{1}=1 / 2, \quad \beta_{3}^{1}=1 / 2, \quad \beta_{4}^{1}=1 / 2, \\
& \beta_{1}^{2}=29 / 2, \quad \beta_{2}^{2}=5 / 2, \quad \beta_{3}^{2}=7 / 2, \quad \beta_{4}^{2}=11 / 2,
\end{aligned}
$$

and therefore implementing it, the cooperation of players will be sustainable. Thus receiving $\beta_{i}^{1}$ at the first stage and $\beta_{i}^{2}$ at the second stage, player $i \in N$ will get $\varphi_{i}\left[v\left(\bar{z}_{1}\right)\right]$ in two stages which is exactly her cooperative payoff prescribed by the Shapley value $\varphi\left[v\left(\bar{z}_{1}\right)\right]$.

\subsection{The Core in Two-Stage Three-Person Game}

Consider three-person-game with pairwise interactions on any network and take as solution the core. Find conditions for strongly time-consistency (Petrosyan, 1995) of core in this game.

Define the core $C(\bar{z}) \subset M_{v}$ in game $\Gamma$ and suppose, that for every $z_{1}, z_{2}$, $C(\bar{z}) \neq \emptyset$. 
Definition 3. The core in game $\Gamma$ is set of imputations $x=\left(x_{1}, \ldots, x_{n}\right)$, which satisfied following conditions:

$$
\sum_{i \in S} x_{i} \geq v\left(\bar{z}_{1} ; S\right)
$$

for all $S \subset N$, and

$$
\sum_{i=1}^{N} x_{i}=v\left(\bar{z}_{1} ; N\right) .
$$

For subgame $\Gamma_{z_{2}}$ we have following values of characteristic function:

$$
\begin{gathered}
v\left(\bar{z}_{2} ; \emptyset\right)=0, v\left(\bar{z}_{2} ;\{1\}\right)=w_{13}^{1}+w_{12}^{1}, v\left(\bar{z}_{2} ;\{2\}\right)=w_{12}^{2}+w_{23}^{2}, v\left(\bar{z}_{2} ;\{3\}\right)=w_{13}^{3}+w_{23}^{3}, \\
v\left(\bar{z}_{2} ;\{12\}\right)=\max _{p, l}\left(a_{p l}^{12}+c_{p l}^{12}\right)+w_{13}^{1}+w_{23}^{2}, \\
v\left(\bar{z}_{2} ;\{13\}\right)=\max _{p, l}\left(a_{p l}^{13}+c_{p l}^{13}\right)+w_{12}^{1}+w_{23}^{3}, \\
v\left(\bar{z}_{2} ;\{23\}\right)=\max _{p, l}\left(a_{p l}^{23}+c_{p l}^{23}\right)+w_{12}^{2}+w_{13}^{3}, \\
v\left(\bar{z}_{2} ; N\right)=\max _{p, l}\left(a_{p l}^{12}+b_{p l}^{21}\right)+\max _{p, l}\left(a_{p l}^{13}+b_{p l}^{31}\right)+\max _{p, l}\left(a_{p l}^{23}+b_{p l}^{32}\right) .
\end{gathered}
$$

Imputation $x$ belongs to the core $C\left(\bar{z}_{2}\right)$, when following inequalities holds:

$$
\left\{\begin{array}{c}
x_{1}+x_{2} \geq v\left(\bar{z}_{2} ;\{12\}\right), \\
x_{1}+x_{3} \geq v\left(\bar{z}_{2} ;\{13\}\right), \\
x_{2}+x_{3} \geq v\left(\bar{z}_{2} ;\{23\}\right), \\
x_{1} \geq v\left(\bar{z}_{2} ;\{1\}\right), \\
x_{2} \geq v\left(\bar{z}_{2} ;\{2\}\right), \\
x_{3} \geq v\left(\bar{z}_{2} ;\{3\}\right), \\
x_{1}+x_{2}+x_{3}=v\left(\bar{z}_{2} ; N\right) .
\end{array}\right.
$$

In view of (37) and values of characteristic function, we have:

$$
\left\{\begin{array}{c}
x_{1}+x_{2} \geq \max _{p l}\left(a_{p l}^{12}+c_{p l}^{12}\right)+w_{23}^{2}+w_{13}^{1}, \\
x_{1}+x_{3} \geq \max _{p l}\left(a_{p l}^{13}+c_{p l}^{13}\right)+w_{12}^{1}+w_{23}^{3} \\
x_{2}+x_{3} \geq \max _{p l}\left(a_{p l}^{23}+c_{p l}^{23}\right)+w_{12}^{2}+w_{13}^{3} \\
x_{1}+x_{2}+x_{3}=v\left(\bar{z}_{2} ; N\right)
\end{array}\right.
$$

To simplify the above expressions, use following notations:

$E_{12}=\max _{p l}\left(a_{p l}^{12}+c_{p l}^{12}\right), D_{1}=w_{23}^{2}+w_{13}^{1}$,

$E_{13}=\max _{p l}\left(a_{p l}^{13}+c_{p l}^{13}\right), D_{2}=w_{12}^{1}+w_{23}^{3}$,

$E_{23}=\max _{p l}\left(a_{p l}^{23}+c_{p l}^{23}\right), D_{3}=w_{12}^{2}+w_{13}^{3}$.

Then system of inequalities (38), which defines the structure of the core $C\left(\bar{z}_{2}\right)$ can be rewritten in following way:

$$
\left\{\begin{array}{c}
x_{1}+x_{2} \geq E_{12}+D_{1}, \\
x_{1}+x_{3} \geq E_{13}+D_{2}, \\
x_{2}+x_{3} \geq E_{23}+D_{3}, \\
x_{1}+x_{2}+x_{3}=v\left(\bar{z}_{2} ; N\right) .
\end{array}\right.
$$


Consider the core $C\left(\overline{z_{1}}\right)$ of two-stage game $\Gamma$

$$
\left\{\begin{array}{c}
x_{1}^{\prime}+x_{2}^{\prime} \geq v\left(\bar{z}_{1} ;\{12\}\right), \\
x_{1}^{\prime}+x_{3}^{\prime} \geq v\left(\bar{z}_{1} ;\{13\}\right), \\
x_{2}^{\prime}+x_{3}^{\prime} \geq v\left(\bar{z}_{1} ;\{23\}\right) \\
x_{1}^{\prime}+x_{2}^{\prime}+x_{3}^{\prime}=v\left(\bar{z}_{1} ; N\right) .
\end{array}\right.
$$

Corresponding to (40) and characteristic function, we have:

$$
\left\{\begin{array}{c}
x_{1}^{\prime}+x_{2}^{\prime} \geq \max _{p l}\left(a_{p l}^{12}+c_{p l}^{12}\right), \\
x_{1}^{\prime}+x_{3}^{\prime} \geq \max _{p l}\left(a_{p l}^{13}+c_{p l}^{13}\right), \\
x_{2}^{\prime}+x_{3}^{\prime} \geq \max _{p l}\left(a_{p l}^{23}+c_{p l}^{23}\right), \\
\left.x_{1}^{\prime}+x_{2}^{\prime}+x_{3}^{\prime}=v\left(\bar{z}_{1} ; N\right)\right) .
\end{array}\right.
$$

Using above notations, we will have followings:

$$
\left\{\begin{aligned}
x_{1}^{\prime}+x_{2}^{\prime} & \geq E_{12}, \\
x_{1}^{\prime}+x_{3}^{\prime} & \geq E_{13}, \\
x_{2}^{\prime}+x_{3}^{\prime} & \geq E_{23}, \\
x_{1}^{\prime}+x_{2}^{\prime}+x_{3}^{\prime} & =v\left(\bar{z}_{1} ; N\right) .
\end{aligned}\right.
$$

\section{Strongly Time-Consistency}

Definition 4. The core $C\left(z_{1}\right)$ is strongly time-consistent in game $\Gamma$ (Petrosyan, 1995), if

1. $C\left(\bar{z}_{1}\right) \neq \emptyset, C\left(\bar{z}_{2}\right) \neq \emptyset$

2. For every imputation $x \in C\left(z_{1}\right)$ there exists such IDP $\beta=\left(\beta_{1}, \beta_{2}\right)$, that $x=$ $\beta_{1}+\beta_{2}$ and

$$
C\left(\bar{z}_{1}\right) \supset \beta_{1} \oplus C\left(\bar{z}_{2}\right) .
$$

Here symbol $\oplus$ defines as $a \in R^{n}, B \subset R^{n}$, then $a \oplus B=\{a+b: b \in B\}$.

In accordance to (42) we get:

$$
\left\{\begin{array}{l}
\beta_{1}^{1}+\beta_{2}^{1}+\beta_{1}^{2}+\beta_{2}^{2} \geq E_{12}, \\
\beta_{1}^{1}+\beta_{2}^{1}+\beta_{1}^{3}+\beta_{2}^{3} \geq E_{13}, \\
\beta_{1}^{2}+\beta_{2}^{2}+\beta_{1}^{3}+\beta_{2}^{3} \geq E_{23} .
\end{array}\right.
$$

For strongly time-consistency these inequalities must satisfies with following additional restrictions:

$$
\left\{\begin{array}{l}
\beta_{2}^{1}+\beta_{2}^{2} \geq E_{12}+D_{1}, \\
\beta_{2}^{1}+\beta_{2}^{3} \geq E_{13}+D_{2}, \\
\beta_{2}^{2}+\beta_{2}^{3} \geq E_{23}+D_{3} .
\end{array}\right.
$$

Fix $\beta_{1}$, then for strongly time-consistency we should fulfil conditions (44) for $\beta_{2}$. And $\beta_{2}$ must satisfy (43). Also, from $v\left(\bar{z}_{2} ; N\right)=v\left(\bar{z}_{1} ; N\right)$, follows $\beta_{1}^{1}+\beta_{1}^{2}+\beta_{1}^{3}=0$. If conditions (43) satisfy under minimal values $\beta_{2}^{1}, \beta_{2}^{2}, \beta_{2}^{3}$ from conditions (44), then they satisfy for all other values. We have:

$$
\left\{\begin{array}{l}
-\beta_{1}^{3}+E_{12}+D_{1} \geq E_{12}, \\
-\beta_{1}^{2}+E_{13}+D_{2} \geq E_{13}, \\
-\beta_{1}^{1}+E_{23}+D_{3} \geq E_{23} .
\end{array}\right.
$$

Thus, we have conditions for strongly time-consistency of the core $C\left(\bar{z}_{1}\right)$ in game $\Gamma$. 
Theorem 4 (Bulgakova and Petrosyan, 2016). Suppose, that following inequalities are fulfilled:

$$
\left\{\begin{array}{l}
\beta_{1}^{3} \leq D_{1} \\
\beta_{1}^{2} \leq D_{2} \\
\beta_{1}^{1} \leq D_{3}
\end{array}\right.
$$

(i.e there exists $\beta_{1}$ which satisfied (4)), then the core $C\left(\bar{z}_{1}\right)$ is strongly time-consistent.

\section{Multistage Games with Pairwise Interactions}

In (Bulgakova and Petrosyan, 2019a), the results obtained for the two-stage games are generalized to the case of multistage games.

Let abstract space $\mathbf{Z}$ is given and called space of states. In every state $z \in \mathbf{Z}$ $n$-person nonzero-sum game $\Gamma(z)$ is determined. This is network game with network $g(z)$, where players are vertexes and edges are connections (as in two-stage case). Game $\Gamma(z)$ is a set of simultaneous pairwise bimatrix games $\left\{\gamma_{i j}(z)\right\}$ between neighbours by network, $i \in N, j \in N, i \neq j$.

Let $i \in N, j \in N, i \neq j$. Then $i$ plays with $j$ in bimatrix game $\gamma_{i j}(z)$ with payoff matrices $A_{i j}(z)$ and $C_{i j}(z)$, for players $i$ and $j$, respectively.

$$
\begin{gathered}
A_{i j}(z)=\left(\begin{array}{cccc}
a_{11}^{i j}(z) & a_{12}^{i j}(z) & \cdots & a_{1 r}^{i j}(z) \\
a_{21}^{i j}(z) & a_{22}^{i j}(z) & \cdots & a_{2 r}^{i j}(z) \\
\vdots & \vdots & \ddots & \vdots \\
a_{m 1}^{i j}(z) & a_{m 2}^{i j}(z) & \cdots & a_{m r}^{i j}(z)
\end{array}\right) \\
C_{i j}(z)=\left(\begin{array}{cccc}
c_{11}^{i j}(z) & c_{12}^{i j}(z) & \cdots & c_{1 r}^{i j}(z) \\
c_{21}^{i j}(z) & c_{22}^{i j}(z) & \cdots & c_{2 r}^{i j}(z) \\
\vdots & \vdots & \ddots & \vdots \\
c_{m 1}^{i j}(z) & c_{m 2}^{i j}(z) & \cdots & c_{m r}^{i j}(z)
\end{array}\right) \\
a_{p q}^{i j}(z) \geq 0, c_{p q}^{i j}(z) \geq 0, \quad p=1, \ldots, m, \quad q=1, \ldots, r, \quad i, j \in N .
\end{gathered}
$$

Constants $m$ and $k$ coincide for all $i$ and $j$. When we have game $\gamma_{j i}(z)$, i.e. player $i$ is the second player, he has payoff matrix $C_{j i}(z)=A_{i j}^{T}(z)$, and player $j$ has matrix $A_{j i}(z)=C_{i j}^{T}(z)$. To simplify the following expressions we suppose, that $m$ and $r$ coincide for all $i$ and $j$ and all $z$.

Consider the strategy of player $i$ in game $\Gamma(z)$. It is a vector $u_{i}(z)=\left(u_{i}^{1}(z), \ldots, u_{i}^{j}(z), \ldots u_{i}^{n}(z)\right)$, where $u_{i}^{j}$ is strategy of player $i$ in bimatrix game $\gamma_{i j}(z)$. So, we have, that strategy of player $i$ is a vector consisting from rows (pure strategies), chosen by him in bimatrix games $\gamma_{i j}(z)$. Denote by $u(z)=\left(u_{1}(z), \ldots, u_{n}(z)\right)$ strategy profile in game $\Gamma(z)$. Strategy of player $j$ is a vector consisting from columns in corresponding bimatrix games $\gamma_{i j}(z)$. Define payoff of player $i$ in game $\Gamma(z)$ in following way:

$$
K_{i}(z)=\sum_{j=1, j \neq i}^{n} a_{u_{i}^{j}(z) u_{j}^{i}(z)}^{i j}(z) .
$$

Consider game $\Gamma(z)$ in cooperative form. As in two-stage case, introduce characteristic function $v(S ; z), \quad S \subset N$ as lower (maxmin) value in two-person zero-sum 
game between $S$ and $N \backslash S$, based on game $\Gamma(z)$

$$
\begin{aligned}
& \omega_{i j}^{i}(z)=\max _{p} \min _{q} a_{p q}^{i j}(z), \quad p=1, \ldots, m ; \quad q=1, \ldots, r, \\
& \omega_{i j}^{j}(z)=\max _{q} \min _{p} c_{p q}^{i j}(z), \quad p=1, \ldots, m ; \quad q=1, \ldots, r .
\end{aligned}
$$

Lemma 1. Characteristic function $v(S ; z)$ is defined in the following way

$$
\begin{gathered}
v(\{\emptyset\} ; z)=0, \\
v(\{i\} ; z)=\sum_{j \in N, j \neq i} \omega_{i j}^{i}(z), \\
v(S ; z)=\frac{1}{2} \sum_{i \in S} \sum_{j \in S, j \neq i} \max _{p, q}\left(a_{p q}^{i j}(z)+c_{p q}^{i j}(z)\right)+\sum_{i \in S} \sum_{j \in N \backslash S} \omega_{i j}^{i}(z), \quad S \subset N, \\
v(N ; z)=\frac{1}{2} \sum_{i \in N} \sum_{j \in N, j \neq i} \max _{p, q}\left(a_{p q}^{i j}(z)+c_{p q}^{i j}(z)\right) .
\end{gathered}
$$

Suppose, that in state $z \in Z$ in game $\Gamma(z)$ players choose the strategy profile: $u_{i}(z)=\left(u_{i}^{1}(z), \ldots, u_{i}^{n}(z)\right)$. Then the game moves to the state $z^{\prime}$, where game $\Gamma\left(z^{\prime}\right)$ takes place, with simultaneous bimatrix games with matrices, depended from strategies chosen on previous state $z$. Thus the state $z^{\prime}$ depends on state $z$ and strategies in this state. We can introduce function $T: \mathbf{Z} \times \prod_{i=1}^{n} U_{i} \rightarrow \mathbf{Z}$ by formula:

$$
z^{\prime}=T\left(z ; u_{1}(z), u_{2}(z), \ldots, u_{n}(z)\right) .
$$

Multistage game $G(z)$ develops in following way. Game $G\left(z_{1}\right)$ starts in state $z_{1}$. In state $z_{1}$ game $\Gamma\left(z_{1}\right)$ is take place, players choose their strategies $u_{1}\left(z_{1}\right), u_{2}\left(z_{1}\right), \ldots$, $u_{n}\left(z_{1}\right)$, then they pass to the state $z_{2}=T\left(z_{1} ; u_{1}\left(z_{1}\right), u_{2}\left(z_{1}\right), \ldots, u_{n}\left(z_{1}\right)\right)$. In state $z_{k}$ players play game $\Gamma\left(z_{k}\right)$, choose strategies $u_{1}\left(z_{k}\right), u_{2}\left(z_{k}\right), \ldots, u_{n}\left(z_{k}\right)$ and pass to the state $z_{k+1}=T\left(z_{k} ; u_{1}\left(z_{k}\right), u_{2}\left(z_{k}\right), \ldots, u_{n}\left(z_{k}\right)\right)$. Game ends on stage $\ell$ in state $z_{\ell}$. Then, after choosing strategies on every stage of game path $z_{1}, z_{2}, \ldots, z_{k}, \ldots, z_{\ell}$ is realized. Strategy in this multistage game $u(\cdot)=u\{(z)\}$, is a set of players' strategies, defined in every state $z \in \mathbf{Z}$. It follows from above, that any strategy profile $u(\cdot)=\left\{u_{1}(\cdot), \ldots, u_{n}(\cdot)\right\}$ defines only one path, and, therefore a payoff for every player, as sum of his payoffs in games, along the realized path.

$$
K_{i}(u(\cdot))=\sum_{k=1}^{\ell} \sum_{j=1, j \neq i}^{n} a_{u_{i}^{j}\left(z_{k}\right) u_{j}^{i}\left(z_{k}\right)}^{i j}\left(z_{k}\right) .
$$

Note, that set of all paths in multistage game $G(z)$ is finite. Hence, the set of states is also finite. Denote this set by $\overline{\mathbf{Z}} \subset \mathbf{Z}$ Consider special case, when $v(N ; z)$ is the same for all $z \in \overline{\mathbf{Z}}$. Introduce function $w(S), \quad S \subset N$ :

$$
w(S)=\max _{z} v(S ; z) .
$$


Define also characteristic function $V\left(S ; z_{k}\right)$ for multistage game $G\left(z_{k}\right)$, which starts in state $z_{k}$. Function $V\left(S ; z_{k}\right)$ can be calculated using the following analogue of Bellman equation:

$$
\begin{gathered}
V\left(S ; z_{k-1}\right)=\max _{u_{i}, i \in S}\left[\min _{u_{j}, j \in N \backslash S}\left(\sum_{i \in S} K_{i}^{z_{k-1}}\left(u_{1}, \ldots u_{n}\right)+V\left(S ; z_{k}\right)\right)\right]= \\
=\max _{u_{i}, i \in S}\left[\min _{u_{j}, j \in N \backslash S}\left(\sum_{i \in S} K_{i}^{z_{k-1}}\left(u_{1}, \ldots u_{n}\right)+V\left(S ; T\left(z_{k-1} ; u\left(z_{k-1}\right)\right)\right)\right)\right] ; \\
V\left(S ; z_{\ell}\right)=v\left(S ; z_{\ell}\right) .
\end{gathered}
$$

Define

$$
W\left(S ; z_{k}\right)=(l-k+1) w(S)
$$

where $\ell$ is number of stages in game $G\left(z_{1}\right)$.

The following inequality takes place (see Petrosyan and Pankratova, 2018):

$$
W\left(S, z_{k}\right) \geq V\left(S, z_{k}\right), \quad S \subset N .
$$

Remind that one-stage game $\Gamma(z)$ is convex and characteristic function $v(S ; z)$, $S \subset N$ is supermodular. So, we have:

$$
v(X \cup Y ; z) \geq v(X ; z)+v(Y ; z)-v(X \cap Y ; z) .
$$

Take the maximum by $z \in \mathbf{Z}$ in the left and right sides of the inequality

$$
\max _{z} v(X \cup Y ; z) \geq \max _{z} v(X ; z)+\max _{z} v(Y ; z)-\max _{z} v(X \cap Y ; z) .
$$

Since $\max _{z} v(S ; z)=w(S)$, we have:

$$
w(X \cup Y) \geq w(X)+w(Y)-w(X \cap Y) .
$$

The function $w(S)$ is supermodular.

Lemma 2. Function $w(S)$ in game $\Gamma(z)$ is supermodular.

\subsection{Optimality Principle}

Define the set of all imputations $M_{W}$ in game $G\left(z_{1}\right)$ as

$$
M_{W}=\left\{x=\left(x_{1}, \ldots, x_{n}\right): \sum_{i=1}^{n} x_{i}=W\left(N ; z_{1}\right), \quad x_{i} \geq W\left(\{i\} ; z_{1}\right), \quad i \in N\right\} .
$$

And by optimality principle any subset of this set $M_{W}$.

Choose in one-stage game $\Gamma(z)$ as optimality principle an analogue of the core - the set $\hat{C}(w(S))$, containing all imputations $x=\left(x_{1}, \ldots, x_{n}\right)$, which satisfy conditions:

$$
\begin{array}{r}
\sum_{i \in S} x_{i} \geq w(S), \quad S \subset N, \\
\sum_{i=1}^{N} x_{i}=w(N) .
\end{array}
$$


In similar way define set $\hat{C}(W(S ; z))$ in multistage game $G(z)$ as set of imputations, which satisfy following conditions:

$$
\begin{gathered}
\sum_{i \in S} x_{i} \geq W(S ; z), \quad S \subset N, \\
\sum_{i=1}^{N} x_{i}=W(N ; z) .
\end{gathered}
$$

Theorem 5. For any $x \in \hat{C}\left(W\left(S ; z_{k}\right)\right), x=\left(x_{1}, \ldots, x_{n}\right)$ and any $k=\overline{1, l}$, the following equality holds:

$$
x_{i}=(\ell-k+1) x_{i}^{\prime}, \quad \text { where } x_{i}^{\prime} \in \hat{C}(w(S)), \quad i=\overline{1, n} .
$$

\subsection{Strongly Time-Consistency}

Suppose, that players chose strategies $\bar{u}(\cdot)=\left(\bar{u}_{1}(\cdot), \ldots, \bar{u}_{n}(\cdot)\right)$, which maximize the total payoff in $G\left(z_{1}\right)$ :

$$
\sum_{i \in N} K_{i}\left(z_{1} ; \bar{u}_{1}, \ldots, \bar{u}_{n}\right)=\max _{u} \sum_{i \in N} K_{i}\left(z_{1} ; u_{1}, \ldots, u_{n}\right) .
$$

The path $\left(\bar{z}_{1}, \bar{z}_{2}, \ldots, \bar{z}_{\ell}\right)$, corresponding to this strategies is called cooperative path $\left(z_{1}=\bar{z}_{1}\right)$. Rewrite definition of IDP (2) for the game under consideration.

Definition 5. Vector $\beta^{i}, i \in N$ is called imputation distribution procedure (IDP) if for $x \in M_{W}$ (see Petrosyan and Danilov, 1979),

$$
x_{i}=\sum_{k=1}^{\ell} \beta_{k}^{i}, \quad i \in N .
$$

Definition 6. Optimality principle $\hat{C}\left(W\left(S ; \bar{z}_{1}\right)\right)$ is strongly time-consistent in game $G\left(\bar{z}_{1}\right)$ (see Petrosyan, 1995), if

1. $\hat{C}\left(W\left(S ; \bar{z}_{k}\right)\right) \neq \emptyset, \quad k=\overline{1, \ell}$

2. For every imputation $x \in \hat{C}\left(W\left(S ; \bar{z}_{1}\right)\right)$ there exists such IDP $\beta=\left(\beta_{1}, \beta_{2}, \ldots, \beta_{\ell}\right)$, such that

$$
\sum_{j=1}^{k} \beta_{j} \oplus \hat{C}\left(W\left(S ; \bar{z}_{k+1}\right)\right) \subset \hat{C}\left(W\left(S ; \bar{z}_{1}\right)\right), \quad k=\overline{1, \ell-1}
$$

Here symbol $\oplus$ means, that if $a \in R^{n}, B \subset R^{n}$, then $a \oplus B=\{a+b: b \in B\}$.

Proposition 4. Optimality principle $\hat{C}\left(W\left(S ; \bar{z}_{1}\right)\right), \quad k=\overline{1, \ell}$ in game $G\left(\bar{z}_{1}\right)$ is strongly time-consistent. 


\subsection{General Case}

Now consider general case, when values of $v(N ; z)$ do not coincide for different $z$. Introduce function $L\left(\bar{z}_{k}\right)$ is joint payoff of maximal coalition $N$ in state $\bar{z}_{k} \in \bar{z}$, where $\bar{z}$ is cooperative path in game $G\left(\bar{z}_{1}\right)$.

$$
L\left(\bar{z}_{k}\right)=\frac{1}{2} \sum_{i \in N, j \in N, i \neq j}\left(a^{i j}\left(\bar{z}_{k}\right)+a^{j i}\left(\bar{z}_{k}\right)\right) .
$$

Suppose, that $w(S)<\min _{\bar{z}_{k}} L\left(\bar{z}_{k}\right), \quad S \neq N$. And consider function $w\left(S ; \bar{z}_{k}\right)$, $w\left(S ; \bar{z}_{k}\right)=w(S), \quad w\left(N, \bar{z}_{k}\right)=L\left(\bar{z}_{k}\right)$. Let $x$ be any imputation in game $G\left(\bar{z}_{1}\right)$. Consider as optimality principle an analogue of the core in game $G\left(\bar{z}_{k}\right)$ called $\hat{C}\left(W\left(S ; \bar{z}_{k}\right)\right)$; the following set

$$
\begin{gathered}
\sum_{i \in S} x_{i} \geq(\ell-k+1) w(S)=W\left(S ; \bar{z}_{k}\right), \quad S \subset N, \quad S \neq N \\
\sum_{i=1}^{N} x_{i}=\sum_{t=k}^{\ell} L\left(\bar{z}_{k}\right)=\hat{W}\left(N ; \bar{z}_{k}\right) .
\end{gathered}
$$

Suppose, that all $\hat{C}\left(W\left(S ; \bar{z}_{k}\right)\right) \neq \emptyset$.

Definition 7. Optimality principle $\hat{C}\left(W\left(S ; \bar{z}_{1}\right)\right)$ is strongly time-consistent in game $G\left(\bar{z}_{1}\right)$, if

1). $\hat{C}\left(W\left(S ; \bar{z}_{k}\right)\right) \neq \emptyset, \quad k=\overline{1, \ell}$

2). For every imputation $x \in \hat{C}\left(W\left(S ; \bar{z}_{1}\right)\right)$ there exists such IDP $\beta=\left(\beta_{1}, \ldots, \beta_{\ell}\right)$, $x=\sum_{j=1}^{\ell} \beta_{j}$ that

$$
\sum_{j=1}^{k} \beta_{t} \oplus \hat{C}\left(W\left(S ; \bar{z}_{k+1}\right)\right) \subset \hat{C}\left(W\left(S ; \bar{z}_{1}\right)\right), \quad k=\overline{1, \ell} .
$$

Proposition 5. Optimality principle $\hat{C}\left(W\left(S ; \bar{z}_{1}\right)\right)$ is strongly time-consistence.

Example Consider an example with $N=3, k=3$, i.e. 3-person three-stage game starting from state $z_{1}$. In state $z_{1}$ we have 6 matrices, 3 matrices of the first type, and 3 of second type. In state $z_{1}$ all bimatrix games take place with matrices of the first type. In state $z_{1}$ every player $i \in N$ choose his strategy $u_{i}\left(z_{1}\right)$. If all $u_{i}^{j}\left(z_{1}\right)=1, i \in N, j \in N \backslash\{i\}$, then players pass to the state $z_{2}$, where they play bimatrix games with same payoff matrices of first type. If at least one of the components $u_{i}^{j}\left(z_{1}\right)=2, i \in N, j \in N \backslash\{i\}$, then in state $z_{2}$ players play bimatrix games with the second type of matrices. Similarly, the transition to the state $z_{3}$ : if all $u_{i}^{j}\left(z_{2}\right)=1, i \in N, j \in N \backslash\{i\}$, then players in state $z_{3}$ use first type of matrices. If at least one of the components $u_{i}^{j}\left(z_{2}\right)=2, i \in N, j \in N \backslash\{i\}$, then in state $z_{3}$ players use matrices of the second type. Matrices of the first type are:

$$
\begin{aligned}
& A_{12}(z)=\left(\begin{array}{ll}
9 & 0 \\
1 & 1
\end{array}\right), \quad C_{12}(z)=\left(\begin{array}{ll}
9 & 1 \\
0 & 1
\end{array}\right), \quad A_{13}(z)=\left(\begin{array}{cc}
10 & 1 \\
2 & 7
\end{array}\right), \\
& C_{13}(z)=\left(\begin{array}{ll}
8 & 0 \\
0 & 7
\end{array}\right), \quad A_{23}(z)=\left(\begin{array}{cc}
16 & 2 \\
1 & 1
\end{array}\right), \quad C_{23}(z)=\left(\begin{array}{cc}
10 & 1 \\
1 & 0
\end{array}\right) \text {. }
\end{aligned}
$$


Matrices of the second type are:

$$
\begin{array}{ccc}
\widetilde{A}_{12}(z)=\left(\begin{array}{cc}
8 & 5 \\
10 & 16
\end{array}\right), & \widetilde{C}_{12}(z)=\left(\begin{array}{cc}
10 & 13 \\
6 & 6
\end{array}\right), & \widetilde{A}_{13}(z)=\left(\begin{array}{ll}
8 & 12 \\
9 & 10
\end{array}\right) \\
\widetilde{C}_{13}(z)=\left(\begin{array}{cc}
10 & 12 \\
11 & 5
\end{array}\right), & \widetilde{A}_{23}(z)=\left(\begin{array}{cc}
8 & 8 \\
13 & 9
\end{array}\right), & \widetilde{C}_{23}(z)=\left(\begin{array}{cc}
12 & 5 \\
6 & 9
\end{array}\right)
\end{array}
$$

In state $z_{1}$ network with complete graph is given To maximize the joint payoff

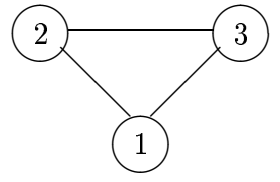

Fig. 3. Network on the first stage

players should to be in touch with all neighbours throughout the game. Strategies of players:

$$
\begin{array}{lll}
u_{1}\left(z_{1}\right)=(0,2,1), & u_{2}\left(z_{1}\right)=(1,0,2), & u_{3}\left(z_{1}\right)=(2,2,0), \\
u_{1}\left(z_{2}\right)=(0,2,1), & u_{2}\left(z_{2}\right)=(2,0,1), & u_{3}\left(z_{2}\right)=(2,1,0), \\
u_{1}\left(z_{3}\right)=(0,2,1), & u_{2}\left(z_{3}\right)=(2,0,1), & u_{3}\left(z_{3}\right)=(2,1,0) .
\end{array}
$$

Calculate the values $\omega_{i j}(z)$ :

$\omega_{12}^{1}\left(z_{1}\right)=1, \quad \omega_{12}^{2}\left(z_{1}\right)=1, \quad \omega_{13}^{1}\left(z_{1}\right)=1, \quad \omega_{13}^{3}\left(z_{1}\right)=0$,

$\omega_{23}^{2}\left(z_{1}\right)=2, \quad \omega_{23}^{3}\left(z_{1}\right)=1$,

$\omega_{12}^{1}\left(z_{2}\right)=10, \quad \omega_{12}^{2}\left(z_{2}\right)=6, \quad \omega_{13}^{1}\left(z_{2}\right)=9, \quad \omega_{13}^{3}\left(z_{2}\right)=10$, $\omega_{23}^{2}\left(z_{2}\right)=9, \quad \omega_{23}^{3}\left(z_{2}\right)=6$.

Values $\omega_{i j}^{i}\left(z_{3}\right)$ will coincide with $\omega_{i j}^{i}\left(z_{2}\right)$ or with $\omega_{i j}^{i}\left(z_{1}\right)$, since there are only two types of matrices in the game.

Calculate the values of characteristic functions $v(S ; z), w\left(S ; z_{k}\right)$ :

\begin{tabular}{|c|c|c|c|c|c|c|c|}
\hline$S$ & $\{1\}$ & $\{2\}$ & $\{3\}$ & $\{12\}$ & $\{13\}$ & $\{23\}$ & $\{123\}$ \\
\hline$v\left(S ; \bar{z}_{1}\right)$ & 2 & 3 & 1 & 21 & 20 & 27 & 62 \\
\hline$v\left(S ; \bar{z}_{2}\right)$ & 19 & 15 & 16 & 40 & 40 & 36 & 66 \\
\hline$v\left(S ; \bar{z}_{3}\right)$ & 19 & 15 & 16 & 40 & 40 & 36 & 66 \\
\hline$w\left(S ; \bar{z}_{1}\right)$ & 19 & 15 & 16 & 40 & 40 & 36 & 58 \\
\hline$w\left(S ; \bar{z}_{2}\right)$ & 19 & 15 & 16 & 40 & 40 & 36 & 66 \\
\hline$w\left(S ; \bar{z}_{3}\right)$ & 19 & 15 & 16 & 40 & 40 & 36 & 66 \\
\hline
\end{tabular}

Game starting from state $z_{1}$, where players choose their strategies and pass to the new state, which depends from this choice. In every state players have only two alternatives: after choosing strategies, pass to the game with first type of payoff matrices, or pass to the second type of payoff matrices.

Numbers 1 and 2 above the arrows on Fig. 4 indicate what type of matrices the players will play in the next state. Calculate the values of function $L$ in nodes $z_{k}$ :

$$
\begin{aligned}
& L\left(z_{1}^{1}\right)=58 L\left(z_{2}^{1}\right)=58 L\left(z_{3}^{1}\right)=62 L\left(z_{2}^{2}\right)=66 L\left(z_{3}^{2}\right)=66 \\
& L\left(z_{3}^{3}\right)=62 L\left(z_{3}^{4}\right)=66
\end{aligned}
$$




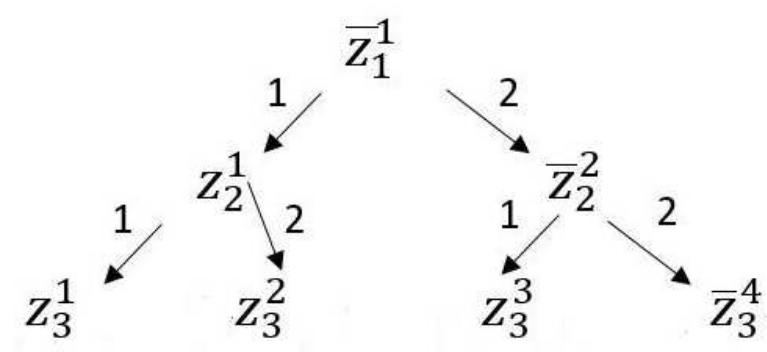

Fig. 4. Tree of all possible states of game.

Cooperative path in game $G\left(z_{1}\right)$ is $\bar{z}=\left(z_{1}^{1}, z_{2}^{2}, z_{3}^{4}\right)=\left(\bar{z}_{1}, \bar{z}_{2}, \bar{z}_{3}\right)$. Calculate the values of characteristic function in multistage game $G\left(z_{1}\right)$ :

\begin{tabular}{|c|c|c|c|c|c|c|c|}
\hline$S$ & $\{1\}$ & $\{2\}$ & $\{3\}$ & $\{12\}$ & $\{13\}$ & $\{23\}$ & $\{123\}$ \\
\hline$V\left(S ; \bar{z}_{3}\right)$ & 19 & 15 & 16 & 40 & 40 & 36 & 66 \\
\hline$V\left(S ; \bar{z}_{2}\right)$ & 38 & 30 & 32 & 80 & 80 & 72 & 132 \\
\hline$V\left(S ; \bar{z}_{1}\right)$ & 40 & 33 & 33 & 101 & 100 & 99 & 190 \\
\hline$W\left(S ; \bar{z}_{3}\right)$ & 19 & 15 & 16 & 40 & 40 & 36 & 66 \\
\hline$W\left(S ; \bar{z}_{2}\right)$ & 38 & 30 & 32 & 80 & 80 & 72 & 132 \\
\hline$W\left(S ; \bar{z}_{1}\right)$ & 57 & 45 & 48 & 120 & 120 & 108 & 190 \\
\hline
\end{tabular}

Condition $w\left(S, z_{k}\right)<\min _{\bar{z}_{k}} L\left(z_{k}\right), \quad S \neq N$ holds:

$$
\max _{S} w\left(S, z_{k}\right)=40<\min _{\bar{z}_{k}} L\left(z_{k}\right)=58 .
$$

Consider an imputation $x \in \hat{C}\left(W\left(S ; \bar{z}_{1}\right)\right)$

$$
\left\{\begin{array}{c}
x_{1} \geq 57 \\
x_{2} \geq 45 \\
x_{3} \geq 48 \\
x_{1}+x_{2} \geq 120 \\
x_{1}+x_{3} \geq 120 \\
x_{2}+x_{3} \geq 108 \\
x_{1}+x_{2}+x_{3}=190 .
\end{array}\right.
$$

And any imputation $\xi \in \hat{C}\left(W\left(S ; \bar{z}_{2}\right)\right)$

$$
\left\{\begin{array}{c}
\xi_{1} \geq 38 \\
\xi_{2} \geq 30 \\
\xi_{3} \geq 32 \\
\xi_{1}+\xi_{2} \geq 80 \\
\xi_{1}+\xi_{3} \geq 80 \\
\xi_{2}+\xi_{3} \geq 72 \\
\xi_{1}+\xi_{2}+\xi_{3}=132
\end{array}\right.
$$

Take as $\beta_{k}$ in IDP $\beta=\left(\beta_{1}, \beta_{2}, \beta_{3}\right)$ an imputation $\alpha \in \hat{C}\left(w\left(S ; \bar{z}_{1}\right)\right),\left(\beta_{k}=\alpha, k=\right.$ $1,2,3)$. 


$$
\left\{\begin{array}{c}
\alpha_{1} \geq 19 \\
\alpha_{2} \geq 15 \\
\alpha_{3} \geq 16 \\
\alpha_{1}+\alpha_{2} \geq 40 \\
\alpha_{1}+\alpha_{3} 40 \\
\alpha_{2}+\alpha_{3} \geq 36 \\
\alpha_{1}+\alpha_{2}+\alpha_{3}=58
\end{array}\right.
$$

Construct the imputation $\hat{x}=\alpha+\xi$

$$
\left\{\begin{array}{c}
\hat{x}_{1} \geq 57 \\
\hat{x}_{2} \geq 45 \\
\hat{x}_{3} \geq 48 \\
\hat{x}_{1}+\hat{x}_{2} \geq 120 \\
\hat{x}_{1}+\hat{x}_{3} \geq 120 \\
\hat{x}_{2}+\hat{x}_{3} \geq 108 \\
\hat{x}_{1}+\hat{x}_{2}+\hat{x}_{3}=190 .
\end{array}\right.
$$

It follows from last inequality, that $\hat{x} \in \hat{C}\left(W\left(S ; \bar{z}_{1}\right)\right)$, which proves strongly timeconsistency of $\hat{C}\left(W\left(S ; \bar{z}_{1}\right)\right)$.

\section{Nonzero-Sum Games with Pairwise Interactions}

In this part we will consider general nonzero-sum game with pairwise interactions. Previously we considered only bimatrix games.

Consider (Bulgakova and Petrosyan, 2019b) multistage nonzero-sum game with finite number of stages. As before, on the first stage players chose their behaviors and formed a network. On next stages simultaneous non-zero sum games will be played. Payoffs depend on controls, chosen on each current stage. Players can change the network on every stage except the first, deleting some connections.

\subsection{The Model}

In every state $z_{k} \in \mathbf{Z}, k>0$ players can change the network by deleting some connections, so we denote the network as $g\left(z_{k}\right)$, to show the network dependency on state.

Denote as $N_{i}\left(g\left(z_{0}\right)\right)$ neighbours of player $i$ in network $g\left(z_{0}\right)$, i. e. $N_{i}\left(g\left(z_{0}\right)\right)=$ $\left\{j \in N \backslash\{i\}: i j \in g\left(z_{0}\right)\right\}$.

When network $g\left(z_{0}\right)$ is formed, the game passed to the state $z_{1}\left(g\left(z_{0}\right)\right)$, which is determined by network $g\left(z_{0}\right)$. In state $z_{1}\left(g\left(z_{0}\right)\right)$ players can delete some connections, which were formed early. So, network $g\left(z_{0}\right)$ in general will change to $g\left(z_{1}\right)$ and we may have new set of neighbours $N_{i}\left(g\left(z_{1}\right)\right)$. On network $g\left(z_{1}\right)$ players play the simultaneous nonzero-sum game $\Gamma\left(z_{1}\right)$.

On the second stage $z_{1}$ player $i, i=\overline{1, n}$, chose control $y_{i}\left(z_{1}\right)=\left(b_{i}\left(z_{1}\right), x_{i}\left(z_{1}\right)\right)$ from set of controls $Y_{i}$, which, unlike the first stage $z_{0}$, contains an additional component $x_{i}\left(z_{1}\right)$ - behavior in game $\Gamma\left(z_{1}\right)$. Where $b_{i}\left(z_{1}\right)$ is vector with components 0 or 1 , defined in following way:

$$
b_{i j}\left(z_{1}\right)=\left\{\begin{array}{l}
1, \text { save connection } i j \\
0, \text { delete connection } i j
\end{array}\right.
$$


i. e. player on second stage can delete some connections but has no abilities to create new connections. Component $x_{i}\left(z_{1}\right)$ of control $y_{i}\left(z_{1}\right)=\left(b_{i}\left(z_{1}\right), x_{i}\left(z_{1}\right)\right)$ is behavior of player $i$ in game $\Gamma\left(z_{1}\right)$ chosen from set $X_{i}\left(z_{1}\right)$, in state $z_{1}$.

Let $y\left(z_{1}\right)=\left(y_{1}\left(z_{1}\right), \ldots, y_{n}\left(z_{1}\right)\right)$ be a strategy profile in game $\Gamma\left(z_{1}\right)$. Player's payoff $i$ in game $\Gamma\left(z_{1}\right)$ is:

$$
H_{i}\left(z_{1}\right)=\sum_{j \in N_{i}\left(g\left(z_{1}\right)\right)} h_{i}\left(y_{i}\left(z_{1}\right), y_{j}\left(z_{1}\right)\right),
$$

where $g\left(z_{1}\right)$ is network, corresponding to strategy profile $y\left(z_{1}\right)$. Functions $h_{i}\left(x_{i}\left(z_{1}\right), x_{j}\left(z_{1}\right)\right) \geq 0$ are given for all $i \in N$ and all pairs $i j$, i. e. all edges of network $g\left(z_{1}\right)$ and all possible states $z \in \mathbf{Z}$.

Players $i \in N$ chose controls $\left(y_{1}\left(z_{k-1}\right), \ldots, y_{n}\left(z_{k-1}\right)\right)$ in state $z_{k-1} \in \mathbf{Z}$ in game $\Gamma\left(z_{k-1}\right)$. Result of this choice is transition to state $z_{k}$, where $\Gamma\left(z_{k}\right)$ is played, with payoffs $h_{i}\left(x_{j}\left(z_{k}\right), x_{i}\left(z_{k}\right)\right)$, depended on controls, chosen in state $z_{k}$. We may defined a transition $T: \mathbf{Z} \times Y_{1} \times Y_{2} \times \ldots \times Y_{n} \rightarrow \mathbf{Z}$ by formula

$$
z_{k}=T\left(z_{k-1} ; y_{1}\left(z_{k-1}\right), y_{2}\left(z_{k-1}\right), \ldots, y_{n}\left(z_{k-1}\right)\right), \quad k=\overline{1, \ell} .
$$

The, function $T$ uniquely defines state $z_{k}$, which follows after state $z_{k-1}$, if players chose controls $y_{1}\left(z_{k-1}\right), y_{2}\left(z_{k-1}\right), \ldots, y_{n}\left(z_{k-1}\right)$.

Consider multistage game $G(z)$, which develops in following way. Game $G\left(z_{0}\right)$ starts in state $z_{0}$. In state $z_{0}$ network $g\left(z_{0}\right)$ is formed, after that players pass to state $z_{1}$. In state $z_{k-1}$ players chose controls $y_{1}\left(z_{k-1}\right), y_{2}\left(z_{k-1}\right), \ldots, y_{n}\left(z_{k-1}\right)$, play game $\Gamma\left(z_{k-1}\right)$ and pass to state $z_{k}=T\left(z_{k-1} ; y_{1}\left(z_{k-1}\right), y_{2}\left(z_{k-1}\right), \ldots, y_{n}\left(z_{k-1}\right)\right)$. Game ends on stage $\ell+1$ in state $z_{\ell}$. Thus, after choosing controls on every state of the game the path $z_{0}, z_{1}, \ldots, z_{k}, \ldots, z_{\ell}$ is realized.

State $z_{k}$ is called acceptable, if there exists the sequence of controls and the sequence of states $z_{0}, z_{1}, \ldots z_{k}, k \leq \ell$ generated by it, defined by formula (63), such that $z_{k}=z$.

Strategy in multistage game: $y_{i}(\cdot), i \in N$, is a rule, which for every acceptable state $z$ associates components $b_{i}(z), x_{i}(z)$ of control in this state, i. e. the choice of connections for deleting, and choice of behavior $x_{i}(z)$ in game $\Gamma(z)$. It the follows from above that any strategy profile $y(\cdot)=\left\{y_{1}(\cdot), \ldots, y_{n}(\cdot)\right\}$ defines only one path in game, and, hence payoff of every player as sum of his payoffs in games, realized along the path.

$$
H_{i}(y(\cdot))=\sum_{k=1}^{\ell} \sum_{j \in N_{i}(g(z))} h_{i}\left(y_{i}\left(z_{k}\right), y_{j}\left(z_{k}\right)\right) .
$$

The set of all paths in game $G(z)$ is finite. So, the set of all acceptable states also is finite. Denote it as $\overline{\mathbf{Z}} \subset \mathbf{Z}$.

Suppose, that players choose controls $\bar{y}_{i}(z), i \in N$, that maximize their joint payoff in game $G(z)$, i. e.

$$
\sum_{k=1}^{\ell} \sum_{i \in N} H_{i}\left(\bar{y}_{1}\left(z_{k}\right), \ldots, \bar{y}_{n}\left(z_{k}\right)\right)=\max _{y} \sum_{k=1}^{\ell} \sum_{i \in N} H_{i}\left(y_{1}\left(z_{k}\right), \ldots, y_{n}\left(z_{k}\right)\right) .
$$

Strategy profile $\bar{y}=\left(\bar{y}_{1}, \ldots, \bar{y}_{n}\right)$ is called cooperative behavior in game $G(z)$, and path, corresponding to controls $\bar{y}_{i}(z), i \in N,\left(\bar{z}_{0}, \bar{z}_{1}, \ldots, \bar{z}_{\ell}\right)$ is called cooperative path $\left(z_{0}=\bar{z}_{0}\right)$. 
Consider one-stage game $\Gamma(z)$ in any state $z \in \mathbf{Z}$ in cooperative form and defined characteristic function $v(S ; z), S \subset N$, for every subset (coalition) $S \subset N$ in following way:

$$
\begin{aligned}
& v(\emptyset ; z)=0, \\
& v(\{i\} ; z)=0, \\
& v(\{i j\} ; z)=\left\{\begin{array}{cc}
h_{i}\left(\bar{x}_{i}(z) ; \bar{x}_{j}(z)\right)+h_{j}\left(\bar{x}_{j}(z) ; \bar{x}_{i}(z)\right), & \text { if } j \in N_{i}(g(z)), \\
0, & \text { other, }
\end{array}\right. \\
& v(S ; z)=\sum_{i \in S} \sum_{j \in N_{i}(g(z)) \cap S} h_{i}\left(\bar{x}_{i}(z) ; \bar{x}_{j}(z)\right), \\
& v(N ; z)=\sum_{i \in N} \sum_{j \in N_{i}(g(z))} h_{i}\left(\bar{x}_{i}(z) ; \bar{x}_{j}(z)\right),
\end{aligned}
$$

where $\bar{x}_{i}(z), \bar{x}_{j}(z)$ calculated corresponding to (64).

We see that to calculate values of characteristic function, we should find cooperative behavior in game $G\left(z_{0}\right)$ and after that calculate $v\left(S ; z_{k}\right), k=\overline{1, \ell}$ as payoffs, under assumption, that players choose cooperative behavior as components of their controls.

Defind the characteristic function $V\left(S ; z_{k}\right)$ in multistage game $G\left(z_{k}\right)$, which starts in state $z_{k}$, as sum of coalition $S$ payoffs along the cooperative path $\left(\bar{y}\left(z_{0}\right), \bar{y}\left(z_{1}\right), \ldots, \bar{y}\left(z_{l}\right)\right)$ in $\ell-k+1$ stages, starting from $k$ :

$$
\begin{gathered}
V\left(S ; z_{k}\right)=\sum_{r=k}^{\ell} v\left(S ; z_{r}\right)=\sum_{r=k}^{\ell} \sum_{i \in S} \sum_{j \in N_{i}\left(g\left(z_{r}\right)\right) \cap S} h_{i}\left(\bar{x}_{i}\left(z_{r}\right), \bar{x}_{j}\left(z_{r}\right)\right), \\
V\left(S ; z_{\ell}\right)=v\left(S ; z_{\ell}\right) .
\end{gathered}
$$

The following theorem holds:

Theorem 6. Characteristic function $v(S ; z)$ in game $\Gamma(z)$ is supermodular.

\subsection{The Shapley Value}

Consider as a solution of game $\Gamma(z)$ the Shapley value $\varphi[v]=\left(\varphi_{1}[v], \ldots, \varphi_{n}[v]\right)$, (15).

Calculate the difference $[v(S ; z)-v(S \backslash\{i\} ; z)]$ :

$$
[v(S ; z)-v(S \backslash\{i\}) ; z]=\sum_{j \in N_{i}(g(z)) \cap S}\left(h_{i}\left(\bar{x}_{i}(z), \bar{x}_{j}(z)\right)+h_{j}\left(\bar{x}_{j}(z), \bar{x}_{i}(z)\right)\right) .
$$

Substitute calculated above values in the formula of the Shapley value (15)

$$
\varphi_{i}[v]=\sum_{S \subseteq N, i \in S} \frac{(|S|-1) !(n-|S|) !}{n !} \sum_{j \in N_{i}(g(z)) \cap S}\left(h_{i}\left(\bar{x}_{i}(z), \bar{x}_{j}(z)\right)+h_{j}\left(\bar{x}_{j}(z), \bar{x}_{i}(z)\right)\right), \quad i \in N .
$$

This formula does not require the calculating the values of characteristic function for all $S \subset N$. To calculate the components of the Shapley value we need to know only the structure of network $g(z)$. 


\subsection{IDP-Core and Strongly Time-Consistency}

As previously $M_{V}$ is set of all imputations $G\left(z_{0}\right)$.

Introduce IDP $\beta_{i}=\left(\beta_{i}^{0}, \ldots, \beta_{i}^{\ell}\right), i \in N$, for imputation $\xi \in I(V)$, which satisfy

$$
\xi_{i}=\sum_{r=0}^{\ell} \beta_{i}^{r}, \quad i \in N
$$

Consider as optimality principle subset of the core for game $G\left(\bar{z}_{k}\right)$ - IDP-core (Wolf, Zakharov and Petrosian, 2017; Petrosian, Gromova and Pogozhev, 2018) $C\left(V\left(S ; \bar{z}_{k}\right)\right)$, i. e. set of IDP, which satisfied following conditions:

$$
\begin{gathered}
\sum_{i \in S} \beta_{i} \geq V\left(S ; \bar{z}_{k}\right)=\sum_{r=k}^{\ell} \sum_{i \in S} \sum_{j \in N_{i}\left(g\left(z_{r}\right)\right) \cap S} h_{i}\left(\bar{x}_{i}\left(z_{r}\right), \bar{x}_{j}\left(z_{r}\right)\right), \quad S \subset N, \quad S \neq N, \\
\sum_{i=1}^{N} \beta_{i}=V\left(N ; \bar{z}_{k}\right)=\sum_{r=k}^{\ell} \sum_{i \in N} \sum_{j \in N_{i}\left(g\left(z_{r}\right)\right) \cap N} h_{i}\left(\bar{x}_{i}\left(z_{r}\right), \bar{x}_{j}\left(z_{r}\right)\right),
\end{gathered}
$$

where $\beta_{i}=\left(\beta_{1}^{i}, \ldots, \beta_{k}^{i}, \ldots, \beta_{\ell}^{i}\right)$ satisfy following:

$$
\begin{gathered}
\sum_{i \in S} \beta_{i}^{k} \geq v\left(S, \bar{z}_{k}\right)=\sum_{i \in S} \sum_{j \in N_{i}\left(g\left(z_{k}\right)\right) \cap S} h_{i}\left(\bar{x}_{i}\left(z_{k}\right), \bar{x}_{j}\left(z_{k}\right)\right), \quad S \subset N, \\
\sum_{i=1}^{N} \beta_{i}^{k}=v\left(N, \bar{z}_{k}\right)=\sum_{i \in N} \sum_{j \in N_{i}\left(g\left(z_{k}\right)\right) \cap N} h_{i}\left(\bar{x}_{i}\left(z_{k}\right), \bar{x}_{j}\left(z_{k}\right)\right) .
\end{gathered}
$$

Suppose, that all $C\left(V\left(S ; \bar{z}_{k}\right)\right) \neq \emptyset$.

Definition 8. Optimality principle $C\left(V\left(S ; \bar{z}_{0}\right)\right) \neq \emptyset$ is strongly time-consistent in game $G\left(\bar{z}_{0}\right)$, if

1) $C\left(V\left(S ; \bar{z}_{k}\right)\right) \neq \emptyset, \quad k=\overline{0, \ell}$

2) For every imputation $x \in C\left(V\left(S ; \bar{z}_{0}\right)\right)$ there exists such IDP $\beta=\left(\beta_{0}, \ldots, \beta_{\ell}\right)$, $\xi=\sum_{r=0}^{\ell} \beta_{j}$, such that

$$
\sum_{r=0}^{k} \beta_{r} \oplus C\left(V\left(S ; \bar{z}_{k+1}\right)\right) \subset C\left(V\left(S ; \bar{z}_{0}\right)\right), \quad k=\overline{0, \ell} .
$$

Here symbol $\oplus$ means, that if $a \in R^{n}, B \subset R^{n}$, then $a \oplus B=\{a+b: b \in B\}$.

Proposition 6. Optimality principle $C\left(V\left(S ; \bar{z}_{0}\right)\right)$ is strongly time-consistent.

\subsection{Example}

This example is similar to one from the previous section. Consider the case with $N=3, \ell=3$, i. e. game consist four stages and starts in state $z_{0}$. In this state sets $M_{i}$ of players, whom player $i$ can offer a connection, are given.

$$
M_{1}=\{2,3\}, \quad M_{2}=\{1,3\}, \quad M_{3}=\{1,2\} .
$$


Restrictions for number of connections for every player are also given.

$$
a_{1}=1, \quad a_{2}=1, \quad a_{3}=2 .
$$

In state $z_{0}$ players choose vectors $b_{i}\left(z_{0}\right)$, formed the network $g\left(z_{0}\right)$ and pass to the state $z_{1}$. In every state $z_{k}, k \geq 1$ players choose controls $y_{i}\left(z_{k}\right)=\left(b_{i}\left(z_{k}\right), x_{i}\left(z_{k}\right)\right)$, where $b_{i}\left(z_{k}\right)$ - player's regulation connections vector (with components 1 and 0 ), and $x_{i}\left(z_{k}\right)$ is equal to

$$
\begin{gathered}
x_{1}\left(z_{k}\right)=x_{1}(z) \in X_{1}=\left\{x_{1}^{1}(z), x_{1}^{2}(z)\right\} \\
x_{2}\left(z_{k}\right)=x_{2}(z) \in X_{2}=\left\{x_{2}^{1}(z), x_{2}^{2}(z)\right\}, \quad x_{3}\left(z_{k}\right)=x_{3}(z) \in X_{3}\left\{x_{3}^{1}(z), x_{3}^{2}(z)\right\}
\end{gathered}
$$

i. e. every player $i$ has the same set of control components $X_{i}$ in every state $z_{k}$.

For all acceptable states $z_{k}, \quad k \geq 1$, and all possible strategies payoffs $h_{i}\left(\bar{x}_{i}\left(z_{r}\right), \bar{x}_{j}\left(z_{r}\right)\right)$ are given in following way: $h_{i}\left(\bar{x}_{i}\left(z_{r}\right), \bar{x}_{j}\left(z_{r}\right)\right)$ and $h_{i}^{\prime}\left(\bar{x}_{i}\left(z_{r}\right), \bar{x}_{j}\left(z_{r}\right)\right)$.

In state $z_{1}$ game takes place with payoffs $h\left(\bar{x}_{i}\left(z_{1}\right), \bar{x}_{j}\left(z_{1}\right)\right)$. In state $z_{1}$ every player $i \in N$ choose his component of control $x_{i}\left(z_{1}\right)$, and if all $x_{i}\left(z_{1}\right)=$ $x_{i}^{1}\left(z_{1}\right), i \in N$, then players pass to the state $z_{2}$, in game, where payoffs are similar $h_{i}\left(\bar{x}_{i}\left(z_{2}\right), \bar{x}_{j}\left(z_{2}\right)\right)$. If at least one of components $x_{i}\left(z_{1}\right)=x_{i}^{2}\left(z_{1}\right), i \in N$, then in state $z_{2}$ players play game with payoffs $h_{i}^{\prime}\left(\bar{x}_{i}\left(z_{2}\right), \bar{x}_{j}\left(z_{2}\right)\right)$. Similarly, the pass to the state $z_{3}$ : if all $x_{i}\left(z_{1}\right)=x_{i}^{1}\left(z_{1}\right), i \in N$, then in state $z_{3}$ players use payoffs $h_{i}\left(\bar{x}_{i}\left(z_{3}\right), \bar{x}_{j}\left(z_{3}\right)\right)$, if at least one of the components $x_{i}\left(z_{1}\right)=x_{i}^{2}\left(z_{1}\right), i \in N$, then payoffs are $h_{i}^{\prime}\left(\bar{x}_{i}\left(z_{3}\right), \bar{x}_{j}\left(z_{3}\right)\right)$.

Payoffs $h_{i}\left(\bar{x}_{i}\left(z_{r}\right), \bar{x}_{j}\left(z_{r}\right)\right)$ :

$$
\begin{aligned}
& h_{1}\left(\bar{x}_{1}^{1}, \bar{x}_{2}^{1}\right)=4, h_{1}\left(\bar{x}_{1}^{1}, \bar{x}_{3}^{1}\right)=5, h_{2}\left(\bar{x}_{2}^{1}, \bar{x}_{3}^{1}\right)=5, \\
& h_{1}\left(\bar{x}_{1}^{2}, \bar{x}_{2}^{1}\right)=3, h_{1}\left(\bar{x}_{1}^{2}, \bar{x}_{3}^{1}\right)=3, h_{2}\left(\bar{x}_{2}^{2}, \bar{x}_{3}^{1}\right)=1, \\
& h_{1}\left(\bar{x}_{1}^{1}, \bar{x}_{2}^{2}\right)=5, h_{1}\left(\bar{x}_{1}^{1}, \bar{x}_{3}^{2}\right)=1, h_{2}\left(\bar{x}_{2}^{1}, \bar{x}_{3}^{2}\right)=4, \\
& h_{1}\left(\bar{x}_{1}^{2}, \bar{x}_{2}^{2}\right)=5, h_{1}\left(\bar{x}_{1}^{2}, \bar{x}_{3}^{2}\right)=2, h_{2}\left(\bar{x}_{2}^{2}, \bar{x}_{3}^{2}\right)=1, \\
& h_{2}\left(\bar{x}_{2}^{1}, \bar{x}_{1}^{1}\right)=4, h_{3}\left(\bar{x}_{3}^{1}, \bar{x}_{1}^{1}\right)=5, h_{3}\left(\bar{x}_{3}^{1}, \bar{x}_{2}^{1}\right)=5, \\
& h_{2}\left(\bar{x}_{2}^{1}, \bar{x}_{1}^{2}\right)=3, h_{3}\left(\bar{x}_{3}^{1}, \bar{x}_{1}^{2}\right)=3, h_{3}\left(\bar{x}_{3}^{1}, \bar{x}_{2}^{2}\right)=1, \\
& h_{2}\left(\bar{x}_{2}^{2}, \bar{x}_{1}^{1}\right)=5, h_{3}\left(\bar{x}_{3}^{2}, \bar{x}_{1}^{1}\right)=1, h_{3}\left(\bar{x}_{3}^{2}, \bar{x}_{2}^{1}\right)=4, \\
& h_{2}\left(\bar{x}_{2}^{2}, \bar{x}_{1}^{2}\right)=5, h_{3}\left(\bar{x}_{3}^{2}, \bar{x}_{1}^{2}\right)=2, h_{3}\left(\bar{x}_{3}^{2}, \bar{x}_{2}^{2}\right)=1 ;
\end{aligned}
$$

payoffs $h_{i}^{\prime}\left(\bar{x}_{i}\left(z_{r}\right), \bar{x}_{j}\left(z_{r}\right)\right)$ :

$$
\begin{aligned}
& h_{1}^{\prime}\left(\bar{x}_{1}^{1}, \bar{x}_{2}^{1}\right)=8, h_{1}^{\prime}\left(\bar{x}_{1}^{1}, \bar{x}_{3}^{1}\right)=6, h_{2}^{\prime}\left(\bar{x}_{2}^{1}, \bar{x}_{3}^{1}\right)=12, \\
& h_{2}^{\prime}\left(\bar{x}_{1}^{2}, \bar{x}_{2}^{1}\right)=3, h_{1}^{\prime}\left(\bar{x}_{1}^{2}, \bar{x}_{3}^{1}\right)=5, h_{2}^{\prime}\left(\bar{x}_{2}^{2}, \bar{x}_{3}^{1}\right)=10, \\
& h_{1}^{\prime}\left(\bar{x}_{1}^{1}, \bar{x}_{2}^{2}\right)=7, h_{1}^{\prime}\left(\bar{x}_{1}^{1}, \bar{x}_{3}^{2}\right)=4, h_{2}^{\prime}\left(\bar{x}_{2}^{1}, \bar{x}_{3}^{2}\right)=5, \\
& h_{1}^{\prime}\left(\bar{x}_{1}^{2}, \bar{x}_{2}^{2}\right)=4, h_{1}^{\prime}\left(\bar{x}_{1}^{2}, \bar{x}_{3}^{2}\right)=3, h_{2}^{\prime}\left(\bar{x}_{2}^{2}, \bar{x}_{3}^{2}\right)=4, \\
& h_{2}^{\prime}\left(\bar{x}_{2}^{1}, \bar{x}_{1}^{1}\right)=8, h_{3}^{\prime}\left(\bar{x}_{3}^{1}, \bar{x}_{1}^{1}\right)=6, h_{3}^{\prime}\left(\bar{x}_{3}^{1}, \bar{x}_{2}^{1}\right)=12, \\
& h_{2}^{\prime}\left(\bar{x}_{2}^{1}, \bar{x}_{1}^{2}\right)=3, h_{3}^{\prime}\left(\bar{x}_{3}^{1}, \bar{x}_{1}^{2}\right)=5, h_{3}^{\prime}\left(\bar{x}_{3}^{1}, \bar{x}_{2}^{2}\right)=10, \\
& h_{2}^{\prime}\left(\bar{x}_{2}^{2}, \bar{x}_{1}^{1}\right)=7, h_{3}^{\prime}\left(\bar{x}_{3}^{2}, \bar{x}_{1}^{1}\right)=4, h_{3}^{\prime}\left(\bar{x}_{3}^{2}, \bar{x}_{2}^{1}\right)=5, \\
& h_{2}^{\prime}\left(\bar{x}_{2}^{2}, \bar{x}_{1}^{2}\right)=4, h_{3}^{\prime}\left(\bar{x}_{3}^{2}, \bar{x}_{1}^{2}\right)=3, h_{3}^{\prime}\left(\bar{x}_{3}^{2}, \bar{x}_{2}^{2}\right)=4 .
\end{aligned}
$$

In state $z_{0}$ players choose their behavior to maximize the joint payoff of all players:

$$
b_{1}\left(z_{0}\right)=(0,0,1), \quad b_{2}\left(z_{0}\right)=(0,0,1), \quad b_{3}\left(z_{0}\right)=(1,1,0) .
$$

As result we have the following network: 


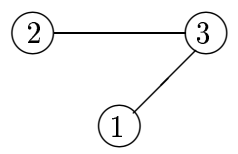

Fig. 5. Network on the first stage

Players have an ability to delete connections on all stages, except the first, but to maximize the joint payoff it is beneficial for players to be in touch with all neighbors throughout the game, i. e. $b_{i}\left(z_{0}\right)=b_{i}\left(z_{1}\right)=b_{i}\left(z_{2}\right)=b_{i}\left(z_{3}\right)$, for all $i \in N$. Components of controls $\bar{y}_{i}(z)$ for players:

$$
\begin{array}{lll}
\bar{x}_{1}\left(z_{1}\right)=x_{1}^{2}, & \bar{x}_{2}\left(z_{1}\right)=x_{2}^{1}, & \bar{x}_{3}\left(z_{1}\right)=x_{3}^{1}, \\
\bar{x}_{1}\left(z_{2}\right)=x_{1}^{1}, & \bar{x}_{2}\left(z_{2}\right)=x_{2}^{2}, & \bar{x}_{3}\left(z_{2}\right)=x_{3}^{1}, \\
\bar{x}_{1}\left(z_{3}\right)=x_{1}^{1}, & \bar{x}_{2}\left(z_{3}\right)=x_{2}^{2}, & \bar{x}_{3}\left(z_{3}\right)=x_{3}^{1} .
\end{array}
$$

Calculate the values of characteristic function $v(S ; z)$ in all states on cooperative path, except $z_{0}$, because on the first stage players only form the network and do not get any payoffs.

\begin{tabular}{|c|c|c|c|c|c|c|c|}
\hline$S$ & $\{1\}$ & $\{2\}$ & $\{3\}$ & $\{12\}$ & $\{13\}$ & $\{23\}$ & $\{123\}$ \\
\hline$v\left(S ; \bar{z}_{1}\right)$ & 0 & 0 & 0 & 6 & 6 & 10 & 16 \\
\hline$v\left(S ; \bar{z}_{2}\right)$ & 0 & 0 & 0 & 14 & 12 & 20 & 32 \\
\hline$v\left(S ; \bar{z}_{3}\right)$ & 0 & 0 & 0 & 14 & 12 & 20 & 32 \\
\hline
\end{tabular}

In state $z_{1}$ players choose their controls and pass to the next state, which depends from this choice. In every state every player has only two alternatives: choose controls and pass in state where play game $\Gamma\left(z_{k}\right)$ with payoffs $h_{i}\left(\bar{x}_{i}\left(z_{k}\right), \bar{x}_{j}\left(z_{k}\right)\right)$, or pass to the state with game, where payoffs are $h_{i}^{\prime}\left(\bar{x}_{i}\left(z_{k}\right), \bar{x}_{j}\left(z_{k}\right)\right)$.

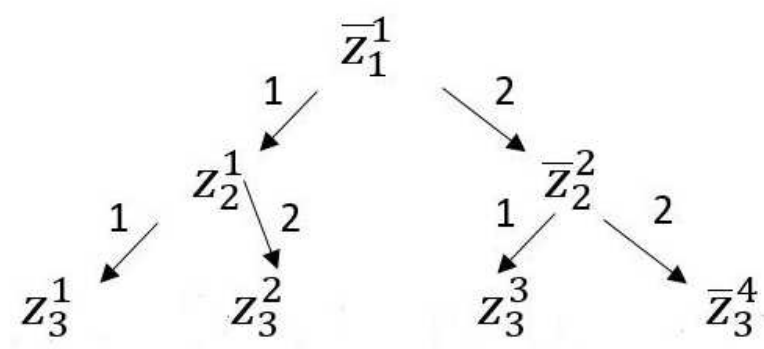

Fig. 6. Tree of all possible states in game

Numbers 1 and 2 above arrows (fig. 6) indicate which payoffs will be used by players in the next state: 1 means $h_{i}\left(\bar{x}_{i}\left(z_{k}\right), \bar{x}_{j}\left(z_{k}\right)\right), 2-h_{i}^{\prime}\left(\bar{x}_{i}\left(z_{k}\right), \bar{x}_{j}\left(z_{k}\right)\right)$.

Cooperative path in game $G\left(z_{0}\right): \bar{z}=\left(z_{0}, z_{1}^{1}, z_{2}^{2}, z_{3}^{4}\right)=\left(\bar{z}_{0}, \bar{z}_{1}, \bar{z}_{2}, \bar{z}_{3}\right)$. Calculate the values of characteristic function in multistage game $G\left(z_{0}\right)$ :

\begin{tabular}{|c|c|c|c|c|c|c|c|}
\hline$S$ & $\{1\}$ & $\{2\}$ & $\{3\}$ & $\{12\}$ & $\{13\}$ & $\{23\}$ & $\{123\}$ \\
\hline$V\left(S ; \bar{z}_{3}\right)$ & 0 & 0 & 0 & 14 & 12 & 20 & 32 \\
\hline$V\left(S ; \bar{z}_{2}\right)$ & 0 & 0 & 0 & 28 & 24 & 40 & 64 \\
\hline$V\left(S ; \bar{z}_{1}\right)$ & 0 & 0 & 0 & 34 & 30 & 50 & 80 \\
\hline
\end{tabular}


Dynamic Cooperative Network Games with Pairwise interactions

Consider an imputation $q \in C\left(V\left(S ; \bar{z}_{1}\right)\right)$ :

$$
\left\{\begin{array}{c}
x_{1} \geq 0 \\
x_{2} \geq 0 \\
x_{3} \geq 0 \\
x_{1}+x_{2} \geq 34 \\
x_{1}+x_{3} \geq 30 \\
x_{2}+x_{3} \geq 50 \\
x_{1}+x_{2}+x_{3}=80 .
\end{array}\right.
$$

As $\beta_{k}$ in IDP $\beta=\left(\beta_{0}, \beta_{1}, \beta_{2}, \beta_{3}\right)$ we will take imputations $\alpha^{k} \in C\left(v\left(S ; \bar{z}_{k}\right)\right)$, which satisfy (70), (71), $\beta_{k}=\alpha, \quad k=1,2,3 ; \beta_{0}$ set equal to zero, and $\beta_{1}$ satisfies the system

$$
\left\{\begin{array}{c}
\alpha_{1}^{1} \geq 0, \\
\alpha_{2}^{1} \geq 0, \\
\alpha_{3} \geq 0, \\
\alpha_{1}^{1}+\alpha_{2}^{1} \geq 6, \\
\alpha_{1}^{1}+\alpha_{3}^{1} \geq 6, \\
\alpha_{2}^{1}+\alpha_{3}^{1} \geq 10, \\
\alpha_{1}^{1}+\alpha_{2}^{1}+\alpha_{3}^{1}=16,
\end{array}\right.
$$

$\beta_{2}$ and $\beta_{3}$ satisfy the inequalities, where $k=2,3$ :

$$
\left\{\begin{array}{c}
\alpha_{1}^{k} \geq 0 \\
\alpha_{2}^{k} \geq 0, \\
\alpha_{3}^{k} \geq 0 \\
\alpha_{1}^{k}+\alpha_{2}^{k} \geq 14, \\
\alpha_{1}^{k}+\alpha_{3}^{k} \geq 12, \\
\alpha_{2}^{k}+\alpha_{3}^{k} \geq 20 \\
\alpha_{1}^{k}+\alpha_{2}^{k}+\alpha_{3}^{k}=32 .
\end{array}\right.
$$

Summing the left and right sides of the inequalities of the last two systems and we get

$$
\left\{\begin{array}{c}
\hat{q}_{1} \geq 0, \\
\hat{q}_{2} \geq 0, \\
\hat{q}_{3} \geq 0 \\
\hat{q}_{1}+\hat{q}_{2} \geq 34, \\
\hat{q}_{1}+\hat{q}_{3} \geq 30, \\
\hat{q}_{2}+\hat{q}_{3} \geq 50, \\
\hat{q}_{1}+\hat{q}_{2}+\hat{q}_{3}=80 .
\end{array}\right.
$$

From last inequality follows, that $\hat{q} \in C\left(V\left(S ; \bar{z}_{0}\right)\right)$. In other words, imputation from set $C\left(V\left(S ; \bar{z}_{0}\right)\right)$, can be decomposed on the sum of imputations from the sets $C\left(v\left(S ; \bar{z}_{k}\right)\right), k=1,2,3$,. This proves strongly time consistency of optimality principle $C\left(V\left(S ; \bar{z}_{0}\right)\right)$.

\section{Conclusion}

The paper discusses the results in the field of cooperative network games with pairwise interaction. Two-stage and multistage games and various approaches to 
the determination of the characteristic function in each of them are considered. The cooperative solutions, such as the Shapley value and the core, are examined and the properties of their time-consistency as well as strongly time-consistency are investigated and the conditions for it are found. Special types of networks are considered, such as a complete network, a star-network, and the possibilities for solutions that give such geometric structures are investigated. All results are illustrated by examples.

Acknowledgements. We acknowledge for the support Russian science foundation (project No.17-11-01079).

\section{References}

Bulgakova, M. and L. Petrosyan (2015). Cooperative network games with pairwise interactions. Math. game th. and app., 4(7), 7-18.

Bulgakova, M. and L. Petrosyan (2016). About Strongly Time-Consistency of Core in the Network Game with Pairwise Interactions. Proc. of 2016 Intern. Conf. "Stability and Oscillations of Nonlinear Control Systems", 157-160.

Bulgakova, M. (2019). Solutions of network games with pairwise interactions. Vestnik of S-Petersb. Univ. App. Math. Comp. Sc. Contr. Proc., 15(1), 147-156.

Bulgakova, M. and Petrosyan, L. (2019). Multistage games with pairwise interactions on full graph. Mathematical game theory and applications, 11(1), 3-20.

Bulgakova, M. and Petrosyan, L. (2019). On the multistage network nonzero-sum game. Vestnik of S-Petersb. Univ. App. Math. Comp. Sc. Contr. Proc., 15(4), 603-615.

Dyer, M. and Mohanaraj, V. (2011). Pairwise-Interaction Games. ICALP, 1, 159-170.

Kuzyutin, D. and M. Nikitina (2017). Time consistent cooperative solutions for multistage games with vector payoffs. Operations Research Letters, 269-274.

Petrosian, O., Gromova, E. and Pogozhev, S. (2018). Strong time-consistent subset of core in cooperative differential games with finite time horizon. Automation and Remote Control, 79(10), 1912-1928.

Petrosyan, L. and Danilov, N. (1979). Stability of solutions of non-zero-sum game with transferable payoffs. Vestnik of Leningrad Univiversity, 1(19), 52-59.

Petrosyan, L. (1995) About new strongly time-consistency solutions in cooperative differential games. Proceedings of the Steklov Institute of Mathematics, 211, 335-340.

Petrosyan, L., Sedakov, A. and Bulgakova, M. (2018). Time-Consistent Solutions for TwoStage Network Games with Pairwise Interactions. Mobile Networks and Applications. https://doi.org/10.1007/s11036-018-1127-7

Petrosyan, L., Sedakov, A. and Bochkarev, A. (2013). Two-stage network games. Mathematical game theory and applications, 5(4), 84-104.

Shapley, L. (1953). A value for $n$-person games. Kuhn HW, Tucker AW (eds) Contributions to the theory of games II. Princeton: Princeton University Press, 307-317.

Shapley, L. (1971). Cores of Convex Games. International Journal of Game Theory, 1, 11-26.

Wolf, D., Zakharov, V. and Petrosian, O. (2018). On the existence of IDP-core in cooperative differential games. Mathematical game theory and applications, 9(4), 18-38.

Pankratova, Y., Petrsoyan, L. (2018). New characteristic function for dynamic multistage games. Vestnik of S-Petersb. Univ. App. Math. Comp. Sc. Contr. Proc., 14(4), 316324 . 